
\title{
Correcting the Hydrogen Diffusion Limitation in Rotating Disk
Electrode Measurements of Hydrogen Evolution Reaction Kinetics
}

(4t阝

Jie Zheng, Yushan Yan, ${ }^{*, \mathrm{z}}$ and Bingjun $\mathrm{Xu}^{\mathrm{z}}$

Department of Chemical and Biomolecular Engineering, Center for Catalytic Science and Technology, University of Delaware, Newark, Delaware 19716, USA

\begin{abstract}
Rotating disk electrode (RDE) method is widely employed in studies on the hydrogen oxidation/evolution reaction (HOR/HER) owing to its well-defined mass transport behaviors. While it is accepted that the measured HOR current is controlled by both the electrode kinetics and the diffusion of $\mathrm{H}_{2}$, HER is typically assumed to be free of diffusion limitation. Here we demonstrate that HER could also be diffusion limited when the electrode kinetics is fast, as evidenced by the rotation speed dependent HER current on Pt in acid $(\mathrm{pH}=1)$ and the overlap of the HER polarization curve with the concentration overpotential curve. The HER diffusion limitation originates from the insufficient mass transport of produced $\mathrm{H}_{2}$ from the electrode surface to the bulk electrolyte and the highly reversible nature of HOR/HER. Kinetic analyses based on HER polarization curves on Pt in acid without correcting for the diffusion limitation could lead to inaccurate Tafel slopes and mechanistic interpretations, and significantly underestimated HER activities. A general data analysis protocol based on the reversible Koutecky-Levich equation is developed to obtain accurate kinetic information of HOR/HER even when electrode kinetics is facile. This new method is compared with other existing methods on $\mathrm{Pt}$ disk electrodes at different $\mathrm{pHs}$ and thin-film electrodes with different Pt loadings.

(C) The Author(s) 2015. Published by ECS. This is an open access article distributed under the terms of the Creative Commons Attribution Non-Commercial No Derivatives 4.0 License (CC BY-NC-ND, http://creativecommons.org/licenses/by-nc-nd/4.0/), which permits non-commercial reuse, distribution, and reproduction in any medium, provided the original work is not changed in any way and is properly cited. For permission for commercial reuse, please email: oa@electrochem.org. [DOI: 10.1149/2.0501514jes] All rights reserved.
\end{abstract}

Manuscript submitted July 30, 2015; revised manuscript received September 30, 2015. Published October 10, 2015.

Hydrogen oxidation reaction ( $\mathrm{HOR}, \mathrm{H}_{2} \rightarrow 2 \mathrm{H}^{+}+2 e$ ) and hydrogen evolution reaction (HER, $2 \mathrm{H}^{+}+2 e \rightarrow \mathrm{H}_{2}$ ) are among the most extensively studied electrochemical reactions for their foundational role in establishing the theoretical framework of electrochemistry, and practical importance in the development of renewable energy conversion devices, such as $\mathrm{H}_{2}$-fueled low temperature fuel cells, and electrolyzers. Kinetics of HOR/HER is not only a key metric in evaluating HOR/HER catalysts, but more importantly, a sensitive probe of the pathway through which the two-electron transfer process occurs on the molecular level.

Rotating disk electrode (RDE) method is widely adopted to quantitatively evaluate the intrinsic kinetics of electrochemical reactions, including HOR/HER ${ }^{1-3}$ and oxygen reduction/evolution reaction (ORR/OER), ${ }^{4,5}$ owing to its well-defined mass transport behaviors that can be rigorously derived from the hydrodynamics. ${ }^{6}$ While ORR activities are typically determined by converting the measured currents at a fixed potential (e.g., $0.9 \mathrm{~V}$ vs. RHE) to kinetic currents due to its sluggish reaction kinetics, ${ }^{7,8}$ exchange current density $\left(\mathrm{i}_{0}\right)$ is the more widely adopted measure of HOR/HER activities. ${ }^{3,9}$ Exchange current density can be determined by either fitting the kinetic current density with the Butler-Volmer equation or extrapolating the Tafel plot to zero overpotential. Accurate measurement of kinetic currents, which is free of the contribution from the mass transport of $\mathrm{H}_{2}$ to and from the electrode, is key to the reliability of both methods. It is generally accepted that HOR can be severely limited by the mass transport of $\mathrm{H}_{2}$ to the electrode surface, as evidenced by the plateau in the polarization curve or the anodic limiting current at large overpotentials. The mass transport limitation of $\mathrm{H}_{2}$ in $\mathrm{HOR}$ is primarily attributed to the limited solubility of $\mathrm{H}_{2}(<1 \mathrm{mmol} / \mathrm{L}$ at room temperature), following the Levich equation ${ }^{6}$ as in Eq. 1,

$$
i_{l}=0.62 n F A D^{2 / 3} \nu^{-1 / 6} c_{0} \omega^{1 / 2}=A B c_{0} \omega^{1 / 2}
$$

where $i_{1}$ is the limiting current, $D$ is the diffusivity of hydrogen in electrolyte, $n$ is the number of electrons transferred in HOR $(n=2)$, A is the area of the electrode, $v$ is the kinematic viscosity of the electrolyte and $\mathrm{c}_{0}$ is the solubility of $\mathrm{H}_{2}$ in electrolyte, $\omega$ is the rotation rate and $B=0.62 n F D^{2 / 3} v^{-1 / 6}$. The kinetic current for thin-film electrode is then calculated using the Koutecky-Levich equation ${ }^{1}$ as

*Electrochemical Society Active Member.

zE-mail: yanys@udel.edu; bxu@udel.edu in Eq. 2,

$$
\frac{1}{i}=\frac{1}{i_{k}}+\frac{1}{i_{l}}=\frac{1}{i_{k}}+\frac{1}{A B c_{0} \omega^{1 / 2}}
$$

where $i$ and $i_{k}$ are the measured current and kinetic current, respectively. In contrast, HER is generally assumed to be free of diffusion limitation and indeed no cathodic limiting current is observed in RDE measurements. The lack of diffusion limitation is attributed to the high concentration of $\mathrm{H}^{+}$or $\mathrm{H}_{2} \mathrm{O}$ in acidic or alkaline electrolyte. Therefore, measured currents, compensated for the ohmic loss, in the HER branch are considered to be kinetic currents. ${ }^{10}$

The RDE method is unable to quantify the HOR activity on Pt in acid due to the combination of mass transport limitation of $\mathrm{H}_{2}$ and fast reaction kinetics. The measured current is dominated by the rate of $\mathrm{H}_{2}$ diffusion to the electrode surface, as evidenced by the overlapping HOR polarization curve and concentration overpotential curve, as defined in Eq. 3,

$$
\eta_{d}=-\frac{R T}{2 F} \ln \left(1-\frac{i_{d}}{i_{l}}\right)
$$

where $\eta_{\mathrm{d}}$ is the diffusion overpotential, $i_{d}$ is the diffusion limited current, $i_{l}$ is the maximum current from polarization curves, $\mathrm{R}$ is the universal gas constant $(8,314 \mathrm{~J} /(\mathrm{mol} \cdot \mathrm{K}))$, $\mathrm{T}$ is the temperature in Kelvin and $\mathrm{F}$ is the Faraday's constant $(96,485 \mathrm{~A} \cdot \mathrm{s} / \mathrm{mol})$. Kinetic currents of HOR on Pt in acid must be determined by methods free of mass transport limitations, such as $\mathrm{H}_{2}$-pump method, ${ }^{9,11,12}$ ultramicroelectrode (UME), ${ }^{13,14}$ scanning electrochemical microscopy (SECM) ${ }^{15,16}$ and floating electrode method. ${ }^{17}$ For example, the exchange current density of $\mathrm{HOR} / \mathrm{HER}$ on $\mathrm{Pt} / \mathrm{C}$ in acid obtained using the $\mathrm{H}_{2}$-pump method in a proton exchange membrane fuel cell (PEMFC) configuration is $\sim 70 \mathrm{~mA} / \mathrm{cm}^{2}{ }_{\mathrm{Pt}}$ at $293 \mathrm{~K}$ (extrapolated from the Arrhenius plot), with the transfer coefficient $(\alpha)$ and Tafel slope determined to be $\sim 0.5$ and $\sim 120 \mathrm{mV} / \mathrm{dec}$, respectively, by fitting the polarization curve with the Butler-Volmer equation. ${ }^{9}$

Several studies of HER on Pt in acid using RDE report Tafel slopes around $30 \mathrm{mV} / \mathrm{dec}$ and exchange current densities on the order of $1 \mathrm{~mA} / \mathrm{cm}^{2}{ }_{\mathrm{Pt}},{ }^{18-23}$ which are almost two orders of magnitude lower than the results obtained from $\mathrm{H}_{2}$-pump measurements. ${ }^{9}$ In this study, we demonstrate that RDE measurements of HER kinetics on Pt are also limited by mass transport of the produced $\mathrm{H}_{2}$ from the electrode surface to the bulk electrolyte when the HER kinetics is facile. This is demonstrated by the overlapping of the HER polarization curve with 
the diffusion overpotential curve and the rotation speed dependence of the HER activity. The inefficient diffusion of $\mathrm{H}_{2}$ formed at the electrode to the bulk electrolyte is proposed to be the origin of HER mass transport limitation. A general data analysis protocol based on Koutecky-Levich equation for reversible reactions is developed to obtain accurate HOR/HER kinetic information. Through comparison with a recently reported method, we show that our proposed approach is equally accurate and more general in extracting kinetic parameters.

\section{Experimental}

Preparation of electrodes and electrolytes. - The polycrystalline Pt disk was polished using $0.05 \mu \mathrm{m}$ alumina polishing suspension (Buehler) to a mirror finish prior to electrochemical testing, and the glassy carbon electrodes were pre-polished similarly before preparing thin-film electrodes. Ink suspension of high surface area carbon supported Pt catalyst was prepared by dispersing Pt/C (46.6 wt\%, Tanaka Kikinzoku International, Inc.) into DI water to achieve a concentration of $0.1 \mathrm{mg}_{\mathrm{Pt}} / \mathrm{mL}$ followed by ultrasonication for $1 \mathrm{~h}$ to obtain a uniform suspension. Thin-film Pt/C electrodes were prepared by pipetting different amounts of ink suspension onto pre-polished glassy carbon electrodes to achieve a final loading of 4 to $40 \mu \mathrm{g}_{\mathrm{Pt}} / \mathrm{cm}^{2}{ }_{\text {disk }}$. $0.1 \mathrm{M}$ $\mathrm{HClO}_{4}, 0.5 \mathrm{M} \mathrm{H}_{2} \mathrm{SO}_{4}$ and $0.2 \mathrm{M} \mathrm{H}_{3} \mathrm{PO}_{4}$ were prepared by diluting $\mathrm{HClO}_{4}$ (67-72\%, TraceSELECT, Sigma Aldrich), $\mathrm{H}_{2} \mathrm{SO}_{4}(95 \%$, TraceSELECT, Sigma Aldrich) and $\mathrm{H}_{3} \mathrm{PO}_{4}(80 \%$, Sigma Aldrich) with DI water. $0.1 \mathrm{M} \mathrm{KOH}$ and $4 \mathrm{M} \mathrm{KOH}$ were prepared from $\mathrm{KOH}$ tablets (85 wt\% and $99.99 \%$ metal trace, Sigma Aldrich). Phosphoric acid/phosphate buffer solutions were prepared by adding different volumes (2 to $10 \mathrm{~mL}$ ) of $4 \mathrm{M} \mathrm{KOH}$ into $100 \mathrm{~mL} 0.2 \mathrm{M} \mathrm{H}_{3} \mathrm{PO}_{4}$.

Electrochemical measurements. - The electrochemical measurements were performed in a glass cell for rotating electrodes (PINE Research Instrumentation), with a double junction silver/silver chloride $(\mathrm{Ag} / \mathrm{AgCl})$ electrode as the reference electrode, a Pt wire as the counter electrode and a $5 \mathrm{~mm}$ diameter polycrystalline Pt disk or a $5 \mathrm{~mm}$ diameter glassy carbon as the working electrode using a multichannel potentiostat (Princeton Applied Research). Cyclic voltammograms $(\mathrm{CV})$ of $\mathrm{Pt}$ disk and $\mathrm{Pt} / \mathrm{C}$ were recorded between $\sim 0.03$ to $1.1 \mathrm{~V}$ vs. RHE in Ar-saturated electrolytes at a scanning rate of $50 \mathrm{mV} / \mathrm{s}$. Hydrogen oxidation/evolution reaction (HOR/HER) polarization curves were obtained by RDE measurement in electrolyte with saturated $\mathrm{H}_{2}$, at a scanning rate of $10 \mathrm{mV} / \mathrm{s}$ (for measurement on $\mathrm{Pt}$ disk) or $1 \mathrm{mV} / \mathrm{s}$ (for measurement on $\mathrm{Pt} / \mathrm{C}$ electrodes) and rotation speeds ranging from $100 \mathrm{rpm}$ to $3600 \mathrm{rpm}$ at r.t. (293 K). All potentials reported in this work were converted to the reversible hydrogen electrode (RHE) scale. Potential ranges for HER scans were chosen such that no excessive $\mathrm{H}_{2}$ bubbling occurred.

Impedance measurements. - The internal resistance was measured after HOR/HER measurement by electrochemical impedance spectroscopy (EIS) from $200 \mathrm{kHz}$ to $100 \mathrm{mHz}$ at open circuit voltage $(\mathrm{OCV})$ and used to obtain internal resistance free (iR-free) potential of the working electrode. The resistances for $0.1 \mathrm{M} \mathrm{HClO}_{4}, 0.5 \mathrm{M}$ $\mathrm{H}_{2} \mathrm{SO}_{4}$ and $0.1 \mathrm{M} \mathrm{KOH}$ at $293 \mathrm{~K}$ are about $25 \Omega, 5 \Omega$ and $40 \Omega$, respectively.

\section{Results and Discussion}

Identification of diffusion limitation in measured HER currents. The HOR/HER polarization curve on Pt disk after internal resistance correction in $0.1 \mathrm{M} \mathrm{HClO}_{4}$ reaches the plateau at $\sim 0.06 \mathrm{~V}$ vs. RHE and completely overlaps with the concentration overpotential curve as defined by Eq. 3 (Figure 1), suggesting that both HOR and HER are completely mass transport controlled. To ensure accurate comparison between the polarization curve and diffusion overpotential curve, iR correction is essential even for highly conductive electrolytes, e.g., $0.1 \mathrm{M} \mathrm{HClO}_{4}(25 \Omega),{ }^{3}$ as evidenced by the significant difference of the polarization curves before and after $\mathrm{iR}$ correction (blue dashed curve and red solid curve in Figure 1, respectively). The lack of iR

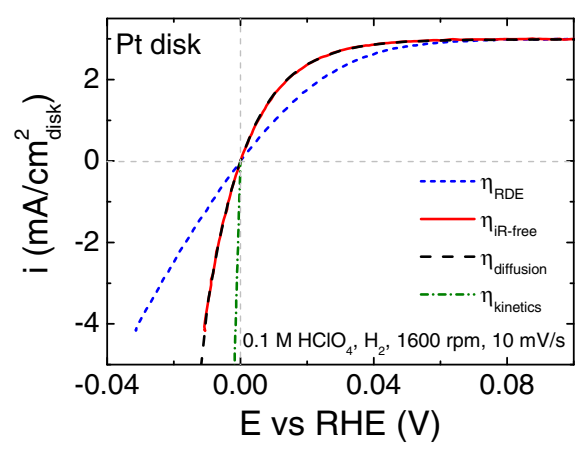

Figure 1. HOR/HER polarization curves on a $\mathrm{Pt}$ disk in $\mathrm{H}_{2}$-saturated $0.1 \mathrm{M}$ $\mathrm{HClO}_{4}$, at a rotation speed of $1600 \mathrm{rpm}$ and a scanning rate of $10 \mathrm{mV} / \mathrm{s}$ before (blue dashed line) and after (red solid line) iR-correction. The black dashed line is the calculated concentration overpotential curve, and the green dot dashed line is the simulated kinetic current using the Butler-Volmer equation (Eq. 4) assuming $\mathrm{i}_{0}=70 \mathrm{~mA} / \mathrm{cm}^{2}$ and $\alpha=0.5$.

correction in several early studies could be a main cause for not recognizing that the current was completely controlled by the mass transport limitation, which in turn led to significant underestimations of the HOR/HER activity on Pt. ${ }^{24,25}$

Measured currents for the highly reversible HOR/HER are the sum of the anodic (positive) and cathodic (negative) currents from HOR and HER, respectively, according to:

$$
i=i_{H O R}+i_{H E R}
$$

Conventionally, the polarization curve at positive overpotentials with positive currents (HOR polarization curve) is referred to as the HOR branch, while the part at negative overpotentials (HER polarization curve) is referred to as the HER branch. It is important to note that HOR and HER occur simultaneously in both branches, albeit with different rates. In this work, we denote the current in the HOR and HER branches as net-HOR and net-HER currents, respectively, to differentiate from the pure HOR and HER currents. The HOR/HER exchange current density $\left(\mathrm{i}_{0}\right)$ at $293 \mathrm{~K}$ on Pt/C and transfer coefficient $(\alpha)$ were determined to be $\sim 70 \mathrm{~mA} / \mathrm{cm}^{2}{ }_{\mathrm{Pt}}$ and $\sim 0.5$ respectively, using the $\mathrm{H}_{2}$ pump method in a PEMFC configuration (with an equivalent $\mathrm{pH}$ of $0)^{9}$ Since no particle size effect has been observed for Pt regarding HOR/HER, ${ }^{3,26}$ the exchange current density and transfer coefficient determined on $\mathrm{Pt} / \mathrm{C}$ can be used to calculate the kinetic current density $\left(i_{k}\right)$ on Pt disk in the HER branch (dashed green line in Figure 1) following the Butler-Volmer equation (Eq. 4),

$$
i_{k}=i_{0}\left[\exp \left(\frac{\alpha F \eta}{R T}\right)-\exp \left(\frac{(\alpha-1) F \eta}{R T}\right)\right]
$$

The measured current in the HER branch should be equal to the kinetic current in the absence of diffusion limitation; however, the calculated kinetic overpotential curve (dashed green line) deviates significantly from the measured polarization curve (solid red line), confirming that the net-HER current is not free of diffusion limitation.

The diffusion current $\left(i_{d}\right)$ is proportional to the limiting current $\left(i_{l}\right)$ at a given potential, according to Eq. 5:

$$
i_{d}=i_{l}\left(1-\exp \left(-\frac{2 F \eta}{R T}\right)\right)
$$

Thus, $i_{d}$ should share the same rotation speed dependence as $i_{l}$ (Eq. 1). It follows that measured currents will also be rotation speed dependent if HER/HOR is completely diffusion limited on Pt disk in acidic media $\left(i_{\text {exp }}=i_{d}\right)$. Indeed, the HOR/HER polarization curves on Pt disk in $0.1 \mathrm{M} \mathrm{HClO}_{4}$ at rotation speeds of $100 \mathrm{rpm}, 400 \mathrm{rpm}, 900 \mathrm{rpm}, 1600$ rpm, $2500 \mathrm{rpm}$ and $3600 \mathrm{rpm}$ match well with their corresponding diffusion overpotential curves in the entire HOR/HER potential window scanned (Figure 2), which is strong evidence that the net-HER current is diffusion limited. Furthermore, a Koutecky - Levich plot at $0.08 \mathrm{~V}$ vs. RHE (Figure 2, inset) yields a straight line passing through 


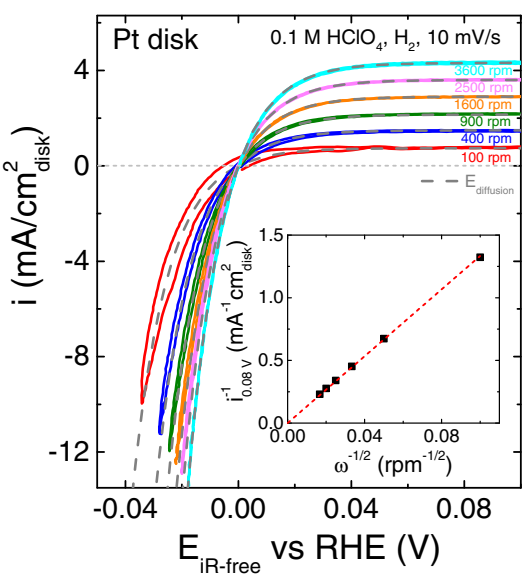

Figure 2. iR-corrected HOR/HER polarization curves at different rotation speeds (100 rpm to $3600 \mathrm{rpm}$ ) on a polycrystalline Pt disk in $\mathrm{H}_{2}$-saturated $0.1 \mathrm{M} \mathrm{HClO}_{4}$ at a scanning rate of $10 \mathrm{mV} / \mathrm{s}$. Dashed gray lines are the diffusion overpotential curves at different rotation speeds. The inset shows a KouteckyLevich plot at $0.08 \mathrm{~V}$ vs. RHE, yielding a straight line with $\mathrm{Bc}_{0}$ value of $0.075 \mathrm{~mA} /\left(\mathrm{cm}^{2}{ }_{\text {disk }} \mathrm{rpm}^{1 / 2}\right)$.

origin, and $\mathrm{a} \mathrm{Bc}_{0}$ value of $0.075 \mathrm{~mA} /\left(\mathrm{cm}^{2}{ }_{\text {disk }} \mathrm{rpm}^{1 / 2}\right)$, in reasonable agreement with the values reported previously. ${ }^{1,3,27}$ Furthermore, the diffusion limited nature of the HER branch on Pt in acid suggests that supersaturation of $\mathrm{H}_{2}$ is likely in the vicinity of the electrode, which provides the driving force of transport $\mathrm{H}_{2}$ produced at the electrode to the bulk. Chen et al. reported that the supersaturation of $\mathrm{H}_{2}$ could be as high as 300 times relative to saturation concentration at room temperature and pressure before nucleation and bubble formation on Pt nanoelectrodes. ${ }^{28,29}$

HER on Pt in base much slower than in acid, and thus HER currents determined by the RDE in base could be kinetics, rather than diffusion, limited. ${ }^{3}$ Indeed, the HOR/HER polarization curves on $\mathrm{Pt} / \mathrm{C}$ in $0.1 \mathrm{M} \mathrm{KOH}$ at rotation speeds from $100 \mathrm{rpm}$ to $3600 \mathrm{rpm}$ deviate significantly from their corresponding concentration overpotential curves (Figure 3), owing to the sluggish kinetics on Pt in the alkaline electrolyte. In addition, the HER polarization curves at different rotation speeds essentially overlap within experimental errors (Figure 3), consistent with a previous report. ${ }^{10}$ The independence of the net-HER current on the rotation speed is also due to the sluggish HER kinet-

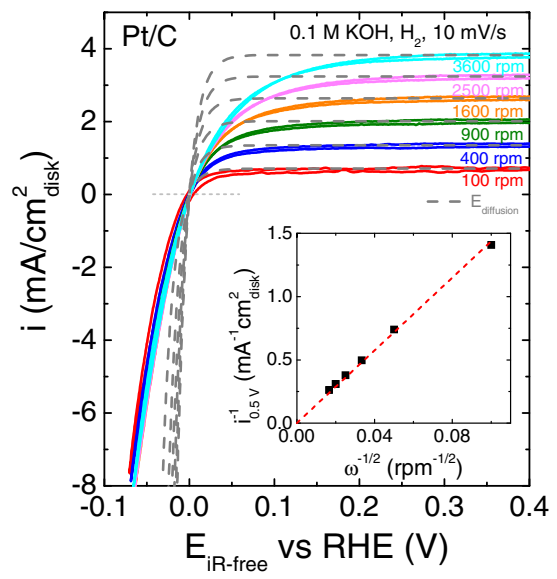

Figure 3. iR-corrected HOR/HER polarization curves at different rotation speeds (100 rpm to $3600 \mathrm{rpm}$ ) on $\mathrm{Pt} / \mathrm{C}$ (loading: $10 \mu \mathrm{g}_{\mathrm{Pt}} / \mathrm{cm}^{2}{ }_{\text {disk }}$ ) in $\mathrm{H}_{2}-$ saturated $0.1 \mathrm{M} \mathrm{KOH}$ at a scanning rate of $10 \mathrm{mV} / \mathrm{s}$. Dashed gray lines are the diffusion overpotential curves at different rotation speeds. The inset shows a Koutecky-Levich plot at $0.5 \mathrm{~V}$ vs. RHE, yielding a straight line with $\mathrm{Bc}_{0}$ value of $0.069 \mathrm{~mA} /\left(\mathrm{cm}^{2}{ }_{\text {disk }} \mathrm{rpm}^{1 / 2}\right)$. ics on Pt in base, which makes the net-HER current kinetics rather than diffusion controlled: the HER overpotential at $-5 \mathrm{~mA} / \mathrm{cm}^{2}{ }_{\text {disk }}$ at $3600 \mathrm{rpm}$ is about $44 \mathrm{mV}$, less than a quarter of which is from $\mathrm{H}_{2}$ mass transport $\left(\eta_{\mathrm{d}}=10 \mathrm{mV}\right)$. Although the kinetic overpotential is dominant when the HER kinetics is sluggish, the contribution of $\eta_{\mathrm{d}}$ in the overall overpotential in the HER branch could still be sizable: The diffusion overpotential $\left(\eta_{\mathrm{d}}\right)$ at $-5 \mathrm{~mA} / \mathrm{cm}^{2}{ }_{\text {disk }}$ increases from $10 \mathrm{mV}$ at the rotation speed of $3600 \mathrm{rpm}$ to $26 \mathrm{mV}$ at 100 $\mathrm{rpm}$. Therefore, a high rotation speed is recommended to facilitate the mass transport of $\mathrm{H}_{2}$ and minimize $\eta_{\mathrm{d}}$ in the RDE measurements of HOR/HER activities. It should be noted that the Levich equation (Eq. 1) does not apply when the rotation speed $(\omega)$ is small, because the hydrodynamic boundary layer becomes large compared with the disk radius, and the assumption used in derivation of the Levich equation no longer holds. ${ }^{30}$ It has been pointed out by Galus et al. that the RDE without rotating will approach unshielded liner diffusion conditions, leading to a finite limiting current. ${ }^{6}$ Indeed, rather than the infinitely large diffusion overpotential and zero net-HER current predicted by the Levich equation, finite net-HER currents are observed when the electrode is not rotating both in $0.1 \mathrm{M} \mathrm{HClO}_{4}$ and $0.1 \mathrm{M} \mathrm{KOH}$ on $\mathrm{Pt}$ (black dashed line in Figure 4). In addition, limiting current densities without rotation in $0.1 \mathrm{M} \mathrm{HClO}_{4}\left(\sim 0.60 \mathrm{~mA} / \mathrm{cm}^{2}{ }_{\text {disk }}\right)$ and in $0.1 \mathrm{M} \mathrm{KOH}\left(0.45 \mathrm{~mA} / \mathrm{cm}^{2}{ }_{\text {disk }}\right)$ are comparable to those obtained at $\omega=100 \mathrm{rpm}$ (Figure 4). Moreover, it is important that constant $\mathrm{H}_{2}$ pressure is maintained throughout the experiment to obtain accurate HER polarization curves even though $\mathrm{H}_{2}$ is not a reactant for HER. Conducting RDE experiments in Ar leads to higher onset potentials for HER ( $\sim 60 \mathrm{mV}$ vs. RHE in both $0.1 \mathrm{M} \mathrm{HClO}_{4}$ and $0.1 \mathrm{M} \mathrm{KOH}$ on a Pt disk) (Figure 5) due to the positive shift of the $\mathrm{H}_{2} / \mathrm{H}^{+}$equilibrium potential at $\mathrm{H}_{2}$ partial pressure less than $1 \mathrm{~atm}$, which explains the "superior" net-HER current in Ar vs. $\mathrm{H}_{2}$ at a rotation speed of $2500 \mathrm{rpm}$. Moreover, the ill-defined equilibrium potential makes it impossible to reveal kinetic information from HER polarization curves obtained in Ar. In $0.1 \mathrm{M} \mathrm{KOH}$, the HER polarization curves measured in $\mathrm{H}_{2}$ and Ar match better than in acid, especially at higher overpotentials $(\sim-25 \mathrm{mV})$ (Figure $5 \mathrm{~b}$ ). This shows that the error caused by the
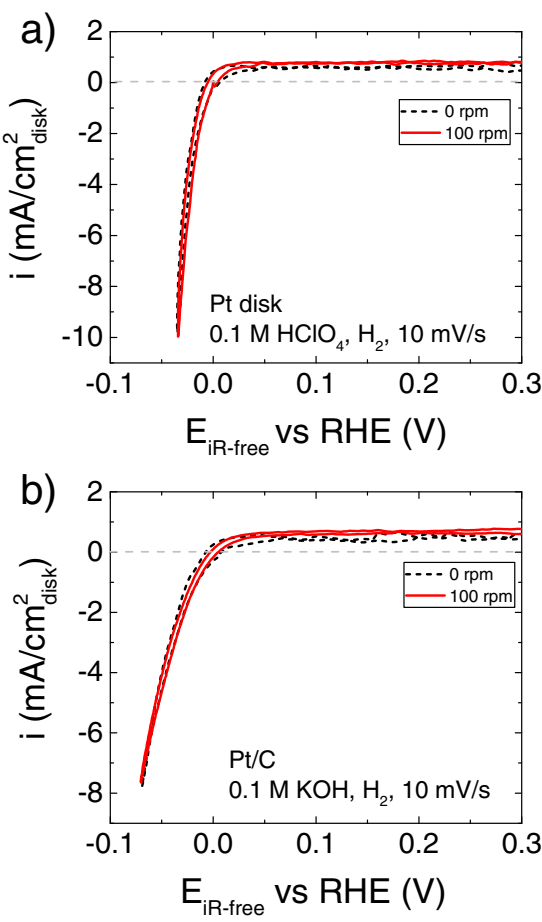

Figure 4. HOR/HER polarization curves on a) $\mathrm{Pt}$ disk in $0.1 \mathrm{M} \mathrm{HClO}_{4}$ and b) $\mathrm{Pt} / \mathrm{C}$ (loading: $10 \mu \mathrm{g}_{\mathrm{Pt}} / \mathrm{cm}^{2}$ disk ) in $0.1 \mathrm{M} \mathrm{KOH}$, in $\mathrm{H}_{2}$ at a scanning rate of $10 \mathrm{mV} / \mathrm{s}$ and rotation speeds of $0 \mathrm{rpm}$ (dash black line) and $100 \mathrm{rpm}$ (solid red line). 

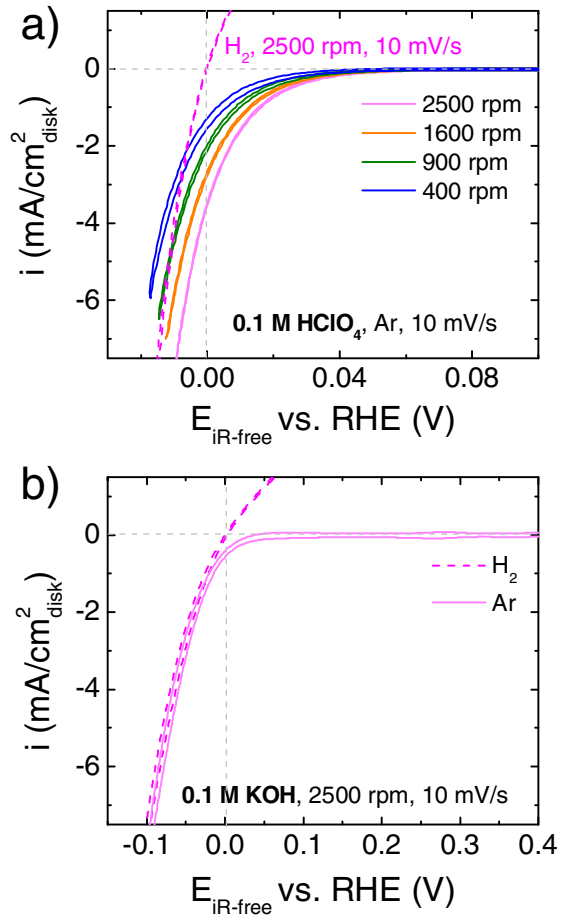

Figure 5. HER polarization curves on Pt disk in a) Ar-saturated $0.1 \mathrm{M} \mathrm{HClO}_{4}$ at a scanning rate of $10 \mathrm{mV} / \mathrm{s}$ at different rotation speeds. Dash magenta line represent HOR/HER polarization curve on Pt disk in $\mathrm{H}_{2}$-saturated 0.1 $\mathrm{M} \mathrm{HClO}_{4}$ at $2500 \mathrm{rpm}$ and $10 \mathrm{mV} / \mathrm{s}$ as comparison, and b) Ar-saturated and $\mathrm{H}_{2}$-saturated $0.1 \mathrm{M} \mathrm{KOH}$ at $2500 \mathrm{rpm}$ and $10 \mathrm{mV} / \mathrm{s}$.

lower $\mathrm{H}_{2}$ partial pressure in HER polarization curves is less when the electrode kinetics is sluggish.

The origin of the diffusion limitation in HER currents. - The highly reversible nature of HOR/HER is key to understanding the diffusion limited behavior of HER. We propose that the diffusion limitation in the HER branch when the electrode kinetics is facile, as in the case Pt electrode in acid, originates from the slow diffusion of the $\mathrm{H}_{2}$ produced on the electrode in HER to the bulk of the electrolyte. The abundance of the reactant, $\mathrm{H}^{+}$, in acid makes the mass transport limitation of the reactant to the electrode unlikely. Meanwhile, the highly reversible nature of HOR/HER makes the efficient transport of product away from the electrode crucial, because the contribution of the pure HOR current to the net-HER current could be significant, especially at low overpotentials. We will discuss only HER in acidic media because the diffusion limitation is more prominent. HER in base $\left(2 \mathrm{H}_{2} \mathrm{O}+2 \mathrm{e} \rightarrow \mathrm{H}_{2}+\mathrm{OH}^{-}\right)$can be considered as a combination of a surface reaction step $\left(2 \mathrm{H}^{+}+2 e \rightarrow \mathrm{H}_{2}\right)$ and a $\mathrm{H}_{2} \mathrm{O}$ dissociation step $\left(2 \mathrm{H}_{2} \mathrm{O} \rightarrow 2 \mathrm{H}^{+}+2 \mathrm{OH}^{-}\right)$, as suggested by Durst et al. ${ }^{12}$ The HOR/HER current can be expressed as follows assuming a first order reaction for $\mathrm{H}_{2}$, and an $\varepsilon$ order reaction for $\mathrm{H}^{+}$,

$$
i=n F A\left(k_{H O R} c_{H_{2}, \text { surf }}-k_{H E R} c_{H^{+}, \text {surf }}\right)
$$

where $k_{H O R}$ and $k_{H E R}$ are the rate constants for HOR and HER, and $c_{\mathrm{H}_{2} \text {,surf }}$ and $c_{\mathrm{H}^{+}, \text {surf }}$ are the surface concentrations of $\mathrm{H}_{2}$ and $\mathrm{H}^{+}$, respectively, and $\varepsilon$ is the reaction order with respect to $\mathrm{H}^{+}$. The assumption of first order reaction with respect to $\mathrm{H}_{2}$ will be justified by our analysis in the following section. In strongly acidic media (Figures 1 and 2), the high concentration of $\mathrm{H}^{+}$makes a steep concentration gradient from bulk to electrode surface unlikely. Therefore, $c_{H^{+}, \text {surf }}$ in the second term of Eq. 6 can be replaced by the bulk $\mathrm{H}^{+}$concentration $\left(c_{H^{+}, \text {bulk }}\right)$, which is independent of rotation speed. In contrast, the slow mass transport of $\mathrm{H}_{2}$ could result in a significant difference in $\mathrm{H}_{2}$ concentrations at the electrode surface, where $\mathrm{H}_{2}$ is produced, and in the bulk electrolyte, especially at low rotation speeds. Since $\mathrm{H}_{2}$ is the reactant of the reverse reaction of HER, i.e., HOR, higher concentration of $\mathrm{H}_{2}$ at the electrode surface leads to larger HOR currents and in turn lower net-HER currents (Figure 2). Surface and bulk $\mathrm{H}_{2}$ concentrations are related through the constraints of mass transport, as Eq. 7,

$$
i=n F A m_{H_{2}}\left(c_{H_{2}, \text { bulk }}-c_{H_{2}, \text { surf }}\right)
$$

where $m_{H_{2}}$ is the mass transport coefficient of $\mathrm{H}_{2}$ and $c_{\mathrm{H}_{2} \text {,bulk }}$ and $c_{H_{2}, \text { surf }}$ are the bulk and surface concentrations of $\mathrm{H}_{2}$, respectively. Substituting the expression for $c_{H_{2}, \text { surf }}$ derived from Eq. 7 into Eq. 6, and assuming $c_{H^{+}, \text {surf }}=c_{H^{+}, \text {bulk }}$,

$$
\begin{aligned}
i= & n F A\left(k_{H O R} c_{H_{2}, \text { bulk }}-k_{H E R} c_{H^{+}, \text {bulk }}^{\varepsilon}\right) /\left(1+\frac{k_{H O R}}{m_{H_{2}}}\right) \\
i_{H O R} & =n F A k_{H O R} c_{H_{2}, \text { surf }} \\
= & n F A k_{H O R}\left[c_{H_{2}, \text { bulk }}+c_{H^{+}, \text {bulk }}^{\varepsilon} \frac{k_{H E R}}{m_{H_{2}}}\right] /\left(1+\frac{k_{H O R}}{m_{H_{2}}}\right)
\end{aligned}
$$

$$
i_{H E R}=n F A k_{H E R} c_{H^{+}, \text {bulk }}^{\varepsilon}
$$

Therefore, the net-HOR/HER and the pure HOR currents are $m_{H_{2}}$ or rotation speed dependent (Eqs. 8-9), because $m_{\mathrm{H}_{2}}$ is proportional to $\omega^{1 / 2}$ according to the Levich equation (Eq. 1). When the rate of $\mathrm{HOR}$ is much smaller than that of $\mathrm{H}_{2}$ diffusion, i.e., $k_{\mathrm{HOR}} \ll m_{\mathrm{H}_{2}}$, Eq. 8 becomes $i=n F A\left(k_{H O R} c_{H_{2}, \text { bulk }}-k_{H E R} c_{H^{+}, \text {bulk }}^{\varepsilon}\right)=i_{k}$, which is consistent with the observation that HER polarization curves on $\mathrm{Pt}$ in base are independent of the rotation speed. Hence, the experimental net-HER currents in base can be considered as kinetic current without introducing significant errors. ${ }^{3,10}$

Impact of diffusion limitation of $\mathrm{H}_{2}$ on determination and interpretation of Tafel slopes. - Correction for internal resistance (iR) is an indispensible step in obtaining reliable Tafel slopes. To illustrate its importance, we chose to present data obtained in $0.5 \mathrm{M}$ $\mathrm{H}_{2} \mathrm{SO}_{4}$ (a typical choice of electrolyte in many HER studies, ${ }^{18,20-23}$ Figure 6a) with an internal resistance of $5 \Omega$ instead of $25 \Omega$ as in the case of $0.1 \mathrm{M} \mathrm{HClO}_{4}$ (Figures 1 and 2). The iR-corrected HER polarization curve on $\mathrm{Pt}$ disk in $\mathrm{H}_{2}$-saturated $0.5 \mathrm{M} \mathrm{H}_{2} \mathrm{SO}_{4}$ at 1600 $\mathrm{rpm}$ and $10 \mathrm{mV} / \mathrm{s}$ deviates significantly from the iR-uncorrected HER polarization curve (Figure 6a). Like in $0.1 \mathrm{M} \mathrm{HClO}_{4}$ (Figure 1), the iR-corrected HER polarization curve in $0.5 \mathrm{M} \mathrm{H}_{2} \mathrm{SO}_{4}$ also almost overlaps with the concentration overpotential curve. The difference in overpotentials obtained with and without iR correction is about $65 \mathrm{mV}$ at $-60 \mathrm{~mA} / \mathrm{cm}^{2}{ }_{\text {disk }}$, demonstrating the importance of iR correction in obtaining accurate kinetic information. The "Tafel slope" of $\mathrm{iR}$-uncorrected HER polarization curve can give rise to a value of $\sim 30 \mathrm{mV} / \mathrm{dec}$ or $306 \mathrm{mV} / \mathrm{dec}$ depending on the choice of overpotential range (from the solid blue line in Figure 6b), both of which are incorrect.

Correction for diffusion limitation also plays an important role in accurate Tafel analysis, especially when the HER kinetics is facile. When the kinetics of HER is sluggish, e.g., in base, the contribution of diffusion overpotential to the overall overpotential is insignificant, and thus the experimental net-HER current can be approximated as the kinetic current. Similar simplification will lead to substantial underestimation of kinetic currents when the rate of HER becomes significant relative to the rate of $\mathrm{H}_{2}$ mass transport. The overlap between the polarization curves and the concentration overpotential curve on $\mathrm{Pt}$ in acid (Figures 2 and 6a) suggests the measured currents are diffusion limited (Eq. 5), i.e., $i_{\text {exp }}=i_{d}$. Thus the apparent Tafel slope determined under this condition $(2.303 \mathrm{RT} / 2 \mathrm{~F}$ or $\sim 30 \mathrm{mV} / \mathrm{dec}$ at $293 \mathrm{~K}$ ) (from solid red line in Figure 6b) reflects the Tafel slope for $\mathrm{H}_{2}$ diffusion, rather than HOR/HER kinetics. Since Tafel slope is frequently used as a diagnostic parameter in mechanistic studies, especially in the identification of the rate-limiting step (RLS), incorrect interpretation of measured Tafel slope could lead to misleading mechanistic interpretations. For 

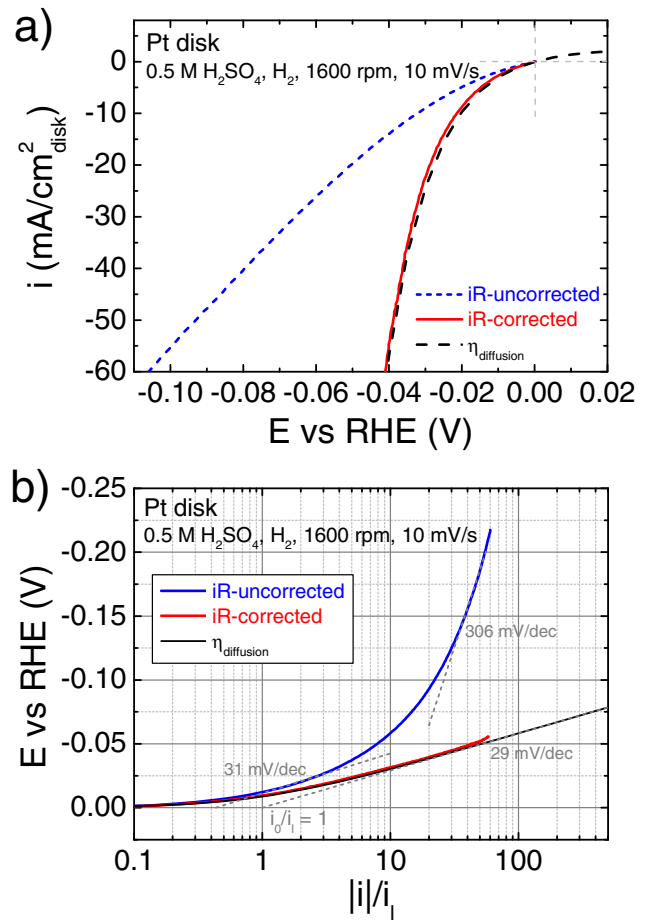

Figure 6. (a) HER polarization curves on $\mathrm{Pt}$ disk in $\mathrm{H}_{2}$ - saturated $0.5 \mathrm{M}$ $\mathrm{H}_{2} \mathrm{SO}_{4}$ at a scanning rate of $10 \mathrm{mV} / \mathrm{s}$, and a rotation speed of $1600 \mathrm{rpm}$ before (blue dash line), after (red line) iR correction and concentration overpotential curve (black dash line), (b) Tafel plot of HER before (blue line) and after (red line) iR correction, black line represents concentration overpotentail curve.

example, a Tafel slope of $30 \mathrm{mV} / \mathrm{dec}$ caused by $\mathrm{H}_{2}$ diffusion limitation, if interpreted as a Tafel slope that reflects the intrinsic HER kinetics, could lead to the conclusion that HER on Pt in acid follow a Tafel-Volmer pathway with Tafel step being the RLS. Using the diffusion-free $\mathrm{H}_{2}$-pump method in a PEMFC configuration, with the equivalent electrolyte $\mathrm{pH}=0$, assuming a transfer coefficient of about 0.5 , a Tafel slope of $120 \mathrm{mV} / \mathrm{dec}(2.303 \mathrm{RT} / \alpha \mathrm{F})$ has been obtained, ${ }^{9,12}$ suggesting that Volmer step is likely the RLS. Since exchange current densities of HER are supposed to be obtained by extrapolating the Tafel slope to $\eta=0 \mathrm{~V}$ vs. RHE, the $30 \mathrm{mV} / \mathrm{dec}$ Tafel slope has led to substantial underestimation of $i_{0}$ reported in the literature. ${ }^{18-23}$ True HER kinetic parameters can only be extracted after the HER branch of the polarization curves are corrected for the overpotential caused by diffusion $\left(\eta_{\mathrm{d}}\right)$, which will be discussed in the next section.

Another important consideration is that Tafel behavior reflects irreversible kinetics, which only occurs at high overpotentials for reversible reactions when the contribution from either the anodic or cathodic reaction to the total current is negligible. It follows that Tafel slopes for reversible reactions, e.g., HOR/HER, should be calculated only at high overpotential regions. Assuming negligible mass transport limitation in the HER branch, the contribution of the pure HOR current drops to less than $1 \%$ to the total current when $\eta<-118 \mathrm{mV}$ at $298 \mathrm{~K}\left(e^{\frac{\alpha F \eta}{R T}} / e^{\frac{(\alpha-1) F \eta}{R T}}=e^{\frac{F \eta}{R T}}<0.01\right)$. For HER entirely controlled by mass transport, as indicated in Eq. 3, Tafel slope no longer reflect the reaction kinetics. However, apparent Tafel slope could be useful for revealing the diffusion limitation. To that end, it is still necessary to draw the Tafel slope at high enough overpotential because the contribution of the pure HOR current is less than $1 \%$ to the total current only when the overpotential is greater than $59 \mathrm{mV}$ at $298 \mathrm{~K}$ $\left(e^{\frac{2 F \eta}{R T}}<0.01\right)$. Tafel slopes determined at overpotentials less than 59 $\mathrm{mV}$ (solid black line in Figure 6b) are not accurate measures of Tafel slopes since the contribution from the reverse reaction to the overall reaction rate is still significant.
Correction of diffusion limitation for both HOR and HER branches. - The contribution of the diffusion current to the net-HOR and net-HER currents must be removed to obtain the kinetic current, based on which true kinetic parameters such as exchange current density and transfer coefficient can be extracted. In this section, we show that the kinetic current can be obtained by the two methods: 1) Extraction of $i_{k}$ from Koutecky-Levich equation for reversible reactions (referred as reversible Koutecky-Levich equation hereafter); and 2) Correction of overpotential and current with the diffusion overpotential and the modified Koutekcy-Levich equation, respectively.

Method 1. reversible koutecky-levich equation.-Since HOR/HER is highly reversible, a reversible Koutecky-Levich equation should be used to calculate kinetic current. For a oneelectron transfer, one-step reversible reaction, $R \leftrightarrow O+e$, a Koutecky-Levich equation for reversible reactions can be written in the form of Eq. 11,

$$
\frac{1}{i}=\frac{1}{i_{k}}+\frac{1}{i_{d}}
$$

where $i_{d}$ is the diffusion limited current defined in Eq. 5 instead of the maximum limiting current $i_{l}{ }^{27,31,32}$ The derivation of the KouteckyLevich equation for reversible reactions is as follows.

The Butler-Volmer equation with mass transport is in the form of Eq. 12 (also see Ref. 30)

$$
\frac{i}{i_{0}}=\left(1-\frac{i}{i_{l, a}}\right) e^{\alpha F \eta / R T}-\left(1-\frac{i}{i_{l, c}}\right) e^{-(1-\alpha) F \eta / R T}
$$

Solving for $\mathrm{i}$, we get

$$
i=\left(e^{\alpha F \eta / R T}-e^{-(1-\alpha) F \eta / R T}\right) /\left(\frac{1}{i_{0}}+\frac{e^{\alpha F \eta / R T}}{i_{l, a}}-\frac{e^{-(1-\alpha) F \eta / R T}}{i_{l, c}}\right)
$$

The kinetic current $i_{k}$ is the current in the absence of mass transport, i.e., $i_{l, a}, i_{l, c} \rightarrow \infty$, thus:

$$
i_{k}=i_{0}\left(e^{\alpha F \eta / R T}-e^{-(1-\alpha) F \eta / R T}\right)
$$

When $\mathrm{i}_{0}$ approaches infinity, the measured current is completely controlled by diffusion, i.e., $i=i_{d}$, and the left hand side of Eq. 12 vanishes:

$$
0=\left(1-\frac{i_{d}}{i_{l, a}}\right) e^{\alpha F \eta / R T}-\left(1-\frac{i_{d}}{i_{l, c}}\right) e^{-(1-\alpha) F \eta / R T}
$$

Solving Eq. 15, we obtain:

$$
i_{d}=\left(1-e^{-F \eta / R T}\right) /\left(\frac{1}{i_{l, a}}-\frac{e^{-F \eta / R T}}{i_{l, c}}\right)
$$

From Eqs. 14 and 16, we get:

$$
\begin{aligned}
\frac{1}{i_{k}}+\frac{1}{i_{d}}= & \frac{1}{i_{0}\left(e^{\alpha F \eta / R T}-e^{-(1-\alpha) F \eta / R T}\right)} \\
& +\left(\frac{1}{i_{l, a}}-\frac{e^{-F \eta / R T}}{i_{l, c}}\right) /\left(1-e^{-F \eta / R T}\right)
\end{aligned}
$$

Eq. 18 can be obtained by taking the reciprocal of the both sides of Eq. 13:

$$
\begin{aligned}
\frac{1}{i}= & \frac{\frac{1}{i_{\mathrm{o}}}+\frac{e^{\alpha F \eta / R T}}{i_{l, a}}-\frac{e^{-(1-\alpha) F \eta / R T}}{i_{l, c}}}{e^{\alpha F \eta / R T}-e^{-(1-\alpha) F \eta / R T}}=\frac{1}{i_{\mathrm{o}}\left(e^{\alpha F \eta / R T}-e^{-(1-\alpha) F \eta / R T}\right)} \\
& +\left(\frac{1}{i_{l, a}}-\frac{e^{-F \eta / R T}}{i_{l, c}}\right) /\left(1-e^{-F \eta / R T}\right)
\end{aligned}
$$

By noting that the right hand sides of Eqs. 17 and 18 are identical, we arrive at Eq. 11.

For a one step, one electron transfer irreversible reaction $(R \rightarrow O+n e)$, we have

$$
\frac{i}{i_{0}}=\left(1-\frac{i}{i_{l, a}}\right) n e^{\alpha F \eta / R T}
$$




$$
\begin{gathered}
i_{k}=i_{0} n e^{\alpha F \eta / R T} \\
i_{d}=i_{l, a}
\end{gathered}
$$

Similar manipulations lead to a Koutecky-Levich equation in the form of Eq. 2, which is widely used to extract kinetic currents from the HOR branch. ${ }^{1-3,10}$ Alternatively, Eq. 2 can be derived by explicitly considering the concentrations of $\mathrm{R}$ at the electrode surface $\left(c_{R, \text { surf }}\right)$ and in the bulk $\left(c_{R, b u l k}\right)$. At steady state, the rate of mass transport of $\mathrm{R}$ should match that of the electrode reaction:

$$
i=n F m_{R}\left(c_{R, \text { bulk }}-c_{R, \text { surf }}\right)
$$

When $c_{R, \text { surf }}$ becomes 0 , the limiting current $\left(i_{l}\right)$ is reached:

$$
i_{l}=n F m_{R} c_{R, b u l k}
$$

where $m_{R}$ is the mass transfer coefficient of R. Hence,

$$
\frac{c_{R, \text { surf }}}{c_{R, \text { bulk }}}=1-\frac{i}{i_{l}}
$$

Since $i=k c_{R, \text { surf }}$ and $i_{k}=k c_{R, b u l k}$,

$$
\frac{i}{i_{k}}=\frac{c_{R, s u r f}}{c_{R, b u l k}}=1-\frac{i}{i_{l}}
$$

which is identical to Eq. 2.

It is critical to recognize that Eqs. 2 and 25 only apply to irreversible reactions (referred to as irreversible Koutecky-Levich equation hereafter), and thus unsuitable for the highly reversible HOR/HER, especially at low overpotentials. ${ }^{14}$ The reversible Koutecky-Levich equation should be employed in extracting kinetic currents of HOR/HER.

If we consider HOR/HER as $\frac{1}{2} H_{2} \leftrightarrow H^{+}+e$ as a one-electron transfer process (not an elementary step), with the apparent reaction orders of $\gamma$ and $\varepsilon$ with respect to $\mathrm{H}_{2}$ and $\mathrm{H}^{+}$, respectively, then

$$
\begin{aligned}
i= & F A k^{0} c_{H_{2}}^{\gamma} \exp \left(\alpha F\left(E-E^{0^{\prime}}\right) / R T\right) \\
& -F A k^{0} c_{H^{+}}^{\varepsilon} \exp \left((\alpha-1) F\left(E-E^{0^{\prime}}\right) / R T\right) \\
i_{0}= & F A k^{0} c_{H_{2}}^{\gamma} \exp \left(\alpha F\left(E_{e q}-E^{0^{\prime}}\right) / R T\right) \\
= & F A k^{0} c_{H^{+}}^{\varepsilon} \exp \left((\alpha-1) F\left(E_{e q}-E^{0^{\prime}}\right) / R T\right)
\end{aligned}
$$

where $E$ is the electrode potential, $E^{0^{\prime}}$ is the formal potential, and $E_{e q}$ is the equilibrium potential of the electrode.

$$
\begin{array}{r}
\text { Since } E_{e q}=E^{0^{\prime}}+\frac{R T}{F} \ln \left(\frac{c_{H+}^{\varepsilon}}{c_{H_{2}}^{\gamma}}\right) \\
i_{0}=F A k^{0} c_{H_{2}}^{\gamma}\left(\frac{c_{H^{+}}^{\varepsilon}}{c_{H_{2}}^{\gamma}}\right)^{\alpha}=F A k^{0} c_{H_{2}}^{(1-\alpha) \gamma} c_{H^{+}}^{\alpha \varepsilon}
\end{array}
$$

From Eq. 29, it is clear that the reaction order for $\mathrm{H}_{2}$ is $(1-\alpha) \gamma$ (with respect to $\mathrm{i}_{0}$ ), which has been determined by Rheinländer et al. ${ }^{10}$ to be 0.5 . Multiple studies have shown that the $\alpha$ value for HOR/HER on Pt is about $0.5,{ }^{3,10,12,31,32}$ which will lead to the conclusion of $\gamma=1$. Therefore, at any given potential, and in turn constant $\mathrm{k}_{\mathrm{HOR}}$ and $\mathrm{k}_{\mathrm{HER}}$, the reaction order of $\mathrm{H}_{2}$ is 1 , consistent with the assumed reaction order value in previous reports. ${ }^{3,12,14}$

Method 2. diffusion overpotential $\left(\eta_{d}\right)$ correction together with current correction. - A recent detailed analysis by Durst et al. points out the importance of correcting the RDE potential by $\eta_{d}$ for HOR branch to obtain the correct kinetic information. ${ }^{9}$ Rheinländer et al. proposed that after $\mathrm{iR}$ and $\eta_{\mathrm{d}}$ corrections for overpotential, a modified Koutecky-Levich equation should be used to calculate kinetic current for the HOR branch based on their observation that the reaction order of hydrogen with respect to the exchange current density is $\frac{1}{2}$, while the HER branch only need to be compensated with iR. ${ }^{10}$ Here we rederive the method of Rheinländer et al. rigorously for a one-electron transfer, one-step reaction below using a different approach. We will show Method 2 is equivalent to Method 1, i.e., using the reversible Koutecky-Levich equation to extract the kinetic current.

For one-electron transfer, one-step reversible reactions, the $i-\eta$, and $\mathrm{i}-\eta_{\mathrm{d}}$ relations can be described by Eq. 12 and Eq. 30 (similar to Eq. 16), respectively.

$$
i=\left(1-e^{-F \eta_{d} / R T}\right) /\left(\frac{1}{i_{l, a}}-\frac{e^{-F \eta_{d} / R T}}{i_{l, c}}\right)
$$

Eq. 30 can be rearranged to $1-\frac{i}{i_{l, a}}=\left(1-\frac{i}{i_{l, c}}\right) e^{-F \eta_{d} / R T}$ and substituted into Eq. 12 to obtain Eq. 31,

$$
\frac{i}{i_{0}}=\left(1-\frac{i}{i_{l, c}}\right)\left(e^{-F \eta_{d} / R T} e^{\alpha F \eta / R T}-e^{-(1-\alpha) F \eta / R T}\right)
$$

Multiply both sides of Eq. 31 by a factor of $e^{(1-\alpha) F \eta_{d} / R T} /\left(1-\frac{i}{i_{l, c}}\right)$,

$$
\frac{i}{i_{0}} e^{(1-\alpha) F \eta_{d} / R T} /\left(1-\frac{i}{i_{l, c}}\right)=e^{\alpha F\left(\eta-\eta_{d}\right) / R T}-e^{-(1-\alpha) F\left(\eta-\eta_{d}\right) / R T}
$$

According to Eq. 30, $e^{-F \eta_{d} / R T}=\frac{1-\frac{i}{i_{l, a}}}{1-\frac{i}{i, c}}$, therefore, $e^{(1-\alpha) F \eta_{d} / R T}=$ $\left(\frac{1-\frac{i}{i, a}}{1-\frac{i}{i l, c}}\right)^{\alpha-1}$, which can be substituted into Eq. 32 to obtain Eq. 33,

$$
\begin{aligned}
i\left(1-\frac{i}{i_{l, a}}\right)^{\alpha-1}\left(1-\frac{i}{i_{l, c}}\right)^{-\alpha} & =i_{0}\left(e^{\alpha F\left(\eta-\eta_{d}\right) / R T}-e^{-(1-\alpha) F\left(\eta-\eta_{d}\right) / R T}\right) \\
& =i_{0}\left(e^{\alpha F \eta_{k} / R T}-e^{-(1-\alpha) F \eta_{k} / R T}\right)=i_{k}
\end{aligned}
$$

The last equality of Eq. 33 indicates an $i_{k}-\eta_{k}$ relation in the form of a Butler-Volmer Equation. The kinetic current $\left(i_{k}\right)$ can be obtained by correcting the measured current (i) via Eq. 34, and kinetic overpotential can be obtained correcting the measured overpotential according to $\eta_{\mathrm{k}}=\eta-\eta_{\mathrm{d}}$

$$
i_{k}=i\left(1-\frac{i}{i_{l, a}}\right)^{\alpha-1}\left(1-\frac{i}{i_{l, c}}\right)^{-\alpha}
$$

For HOR/HER, $i_{l, c}=\infty$ and $\alpha=0.5$, Eq. 34 becomes

$$
\frac{i}{i_{k}}=\left(1-\frac{i}{i_{l, a}}\right)^{0.5}
$$

which is the modified Koutecky-Levich equation proposed by Rheinländer et al. to calculate the kinetic current for HOR branch after $\eta_{\mathrm{d}}$ correction. ${ }^{10}$

It is important to stress that corrections for both the measured current and the overpotential are necessary to obtain the correct $i_{k}-$ $\eta_{\mathrm{k}}$ relation in the form of the Butler-Volmer Equation. If we assume that the uncorrected current (i) relates with the kinetic overpotential $\left(\eta_{\mathrm{k}}\right)$ in the form of the Butler-Volmer Equation,

$$
i=i_{0}\left(e^{\alpha f \eta_{k}}-e^{-(1-\alpha) f \eta_{k}}\right)
$$

By combining Eqs. 31 and 36, at any given $i, \eta, \eta_{\mathrm{k}}$ and $\eta_{\mathrm{d}}$ are related through Eq. 37,

$$
e^{\alpha f \eta_{k}}-e^{-(1-\alpha) f \eta_{k}}=\left(1-\frac{i}{i_{l, c}}\right)\left(e^{f\left(\alpha \eta-\eta_{d}\right)}-e^{-(1-\alpha) f \eta}\right)
$$

rather than the simple expression of $\eta_{\mathrm{k}}=\eta-\eta_{\mathrm{d}}$, which shows the necessity of the correction of the measured current by Eq. 34 .

The procedural differences in the two approaches mentioned above have been summarized in Figure 7. Method 1 is simpler and more general since it does not require the prior knowledge of the $\alpha$ value, and only requires correction for the current, rather than both the current and the potential. 


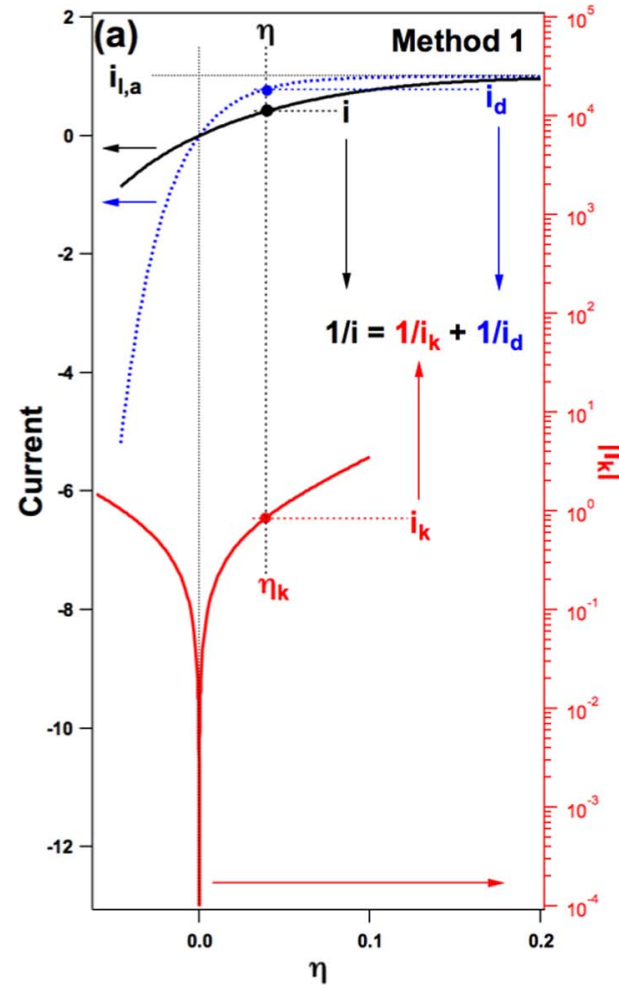

Diffusion limitation correction for HER branch using different approaches. - To illustrate the validity of correcting diffusion limitation in HER using the two methods described in the previous section, HOR/HER polarization curves were obtained on a Pt disk in phosphoric acid/phosphate buffer solution at $\mathrm{pH}=2.4$. These HER curves are close to the concentration overpotential curves and exhibit rotation speed dependence (Figure 8a). The net-HER currents, if not corrected by diffusion, increase as rotation speed increases (solid lines in Figure 8a and dash lines in Figure 8b, 8c). Moreover, the kinetic currents calculated using irreversible Koutecky-Levich equation (Eq. 2) from polarization curves at different rotation speeds in the HOR branch do not overlap (Figure 8b), which confirms that the correction with the irreversible Koutecky-Levich equation is not sufficient. In contrast, both HOR and HER kinetic currents calculated using the reversible Koutecky-Levich equation (Eq. 11) at different rotation speeds overlap, and they are larger than the measured currents (Figure 8c). Furthermore, the overpotential and current corrections by $\eta_{\mathrm{k}}=\eta-\eta_{\mathrm{d}}$ and Eq. 35, respectively, generate almost identical $\left|i_{k}\right|-E-\eta_{d}$ plots at different rotation speeds (Figure 8d), which also match the $\left|i_{k}\right|-E$ plot constructed with the reversible Koutecky-Levich equation (gray dashed line in Figure 8d).
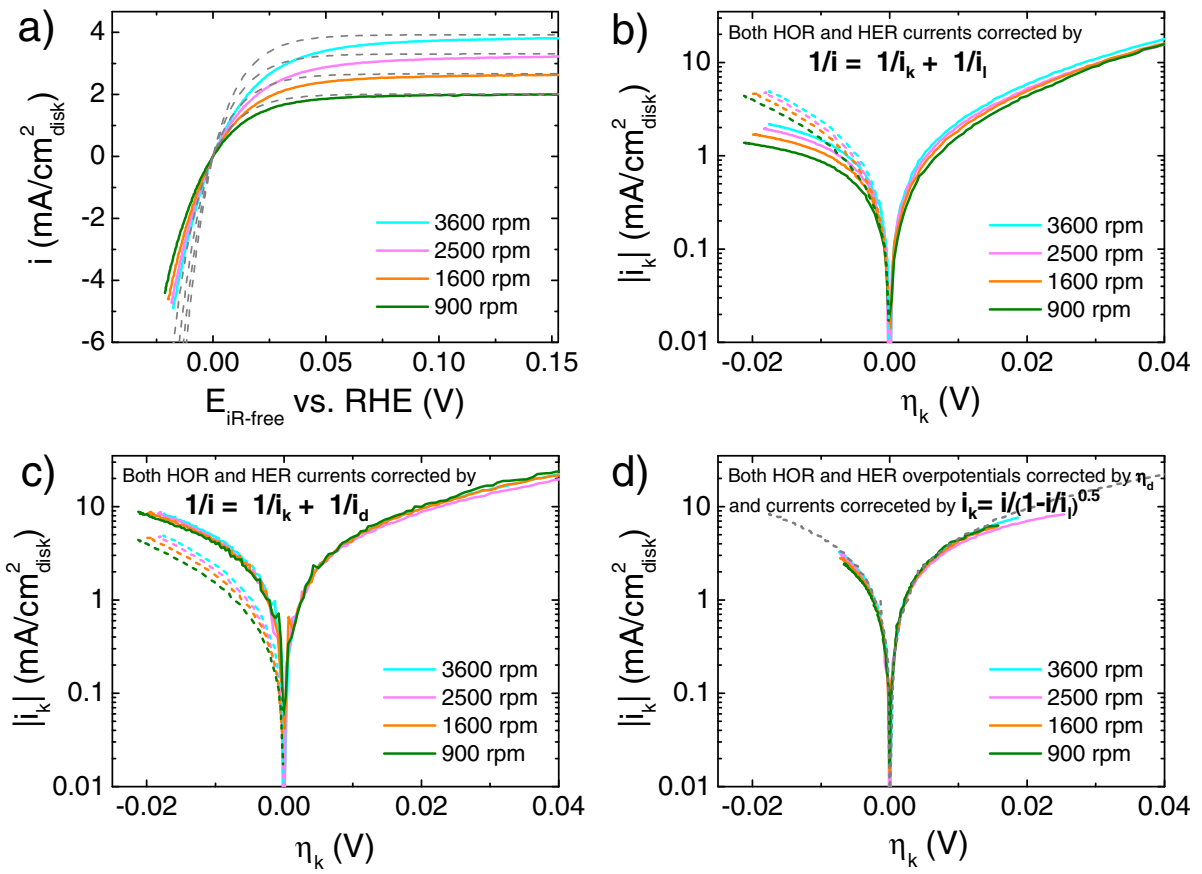

Figure 8. (a) HOR/HER polarization curves on a $\mathrm{Pt}$ disk in $\mathrm{H}_{2}$-saturated phosphoric acid/phosphate buffer solution $(\mathrm{pH}=2.4)$ at $10 \mathrm{mV} / \mathrm{s}$ and rotation speeds from 900 to $3600 \mathrm{rpm}$. Dash lines represent the concentration overpotential curves. (b) Tafel plots of HOR/HER when both HOR and HER kinetic currents were calculated with the irreversible Koutecky-Levich equation (Eq. 2), and the dashed lines represent the experimental net-HER currents, the kinetic overpotential $\eta_{\mathrm{k}}$ is the iR-corrected overpotential ( $E_{i R-f r e e ~} v s . R H E$ ), (c) Tafel plots of HOR/HER when both HOR and HER kinetic currents were calculated with the reversible Koutecky-Levich equation (Eq. 11), and the dashed lines represent the experimental netHER currents, and $\eta_{\mathrm{k}}=\mathrm{E}_{\mathrm{i} R \text {-free }}$ vs. RHE, (d) Tafel plots of HOR/HER when both HOR and HER overpotentials are corrected by diffusion overpotential $\left(\eta_{\mathrm{d}}\right)\left(\eta_{\mathrm{k}}=\mathrm{E}_{\mathrm{iR} \text {-free }}\right.$ vs. RHE $\left.-\eta_{\mathrm{d}}\right)$ and the HOR and HER currents were converted to kinetic current by Eq. 35, the gray dashed line represents the $\left|i_{k}\right|$ vs $\mathrm{E}_{\mathrm{iR} \text {-free }}$ curve in (c) at a rotation speed of $3600 \mathrm{rpm}$ for comparison. 

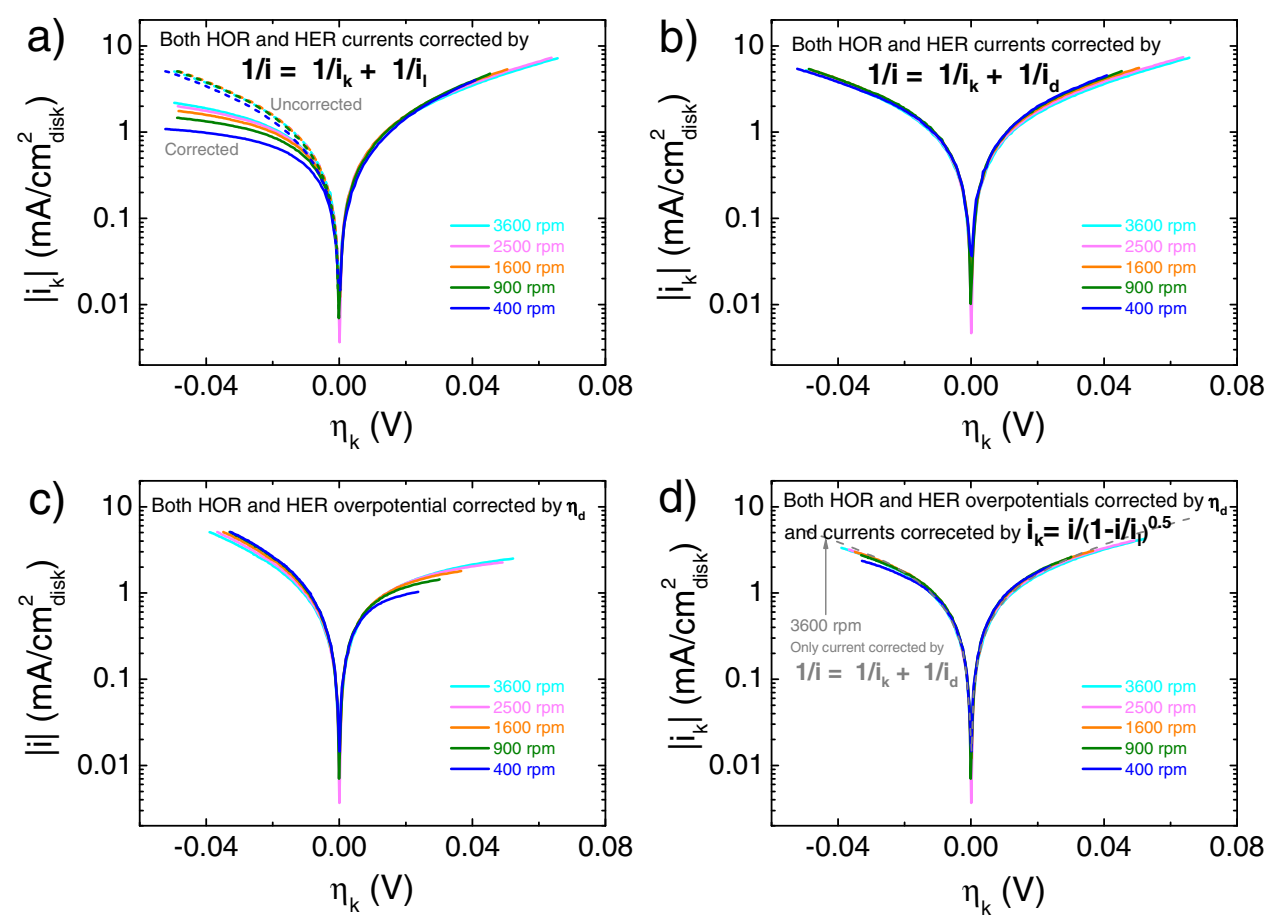

Figure 9. Tafel plots for HOR/HER on Pt/C (loading: $10 \mu \mathrm{g}_{\mathrm{P}} / \mathrm{cm}^{2}$ disk) in $\mathrm{H}_{2}$ saturated $0.1 \mathrm{M} \mathrm{KOH}$ at a scanning rate of $10 \mathrm{mV} / \mathrm{s}$ at different rotation speeds. (a) Both HOR and HER kinetic currents were calculated with the irreversible Koutecky-Levich equation (Eq. 2), dashed lines represents experimental net-HER currents, and $\eta_{\mathrm{k}}=\mathrm{E}_{\mathrm{iR} \text {-free }}$ vs. RHE, (b) Both HOR and HER kinetic currents were calculated with the reversible Koutecky-Levich equation (Eq. 11), and $\eta_{\mathrm{k}}=$ $\mathrm{E}_{\mathrm{iR} \text {-free }}$ vs. RHE, and (c) Both HOR and HER currents were uncorrected while the overpotential is corrected by diffusion overpotential ( $\left.\eta_{\mathrm{d}}\right)$, so $\eta_{\mathrm{k}}=\mathrm{E}_{\mathrm{iR} \text {-free }} \mathrm{vs}$. RHE $-\eta_{\mathrm{d}}$. (d) Both HOR and HER overpotentials are corrected by diffusion overpotential $\left(\eta_{\mathrm{d}}\right)$ and the HOR and HER currents were converted to kinetic current by Eq. $35, \eta_{\mathrm{k}}=\mathrm{E}_{\mathrm{iR} \text {-free }} \mathrm{vs}$. RHE $-\eta_{\mathrm{d}}$, and gray dashed line represents the $\left|\mathrm{i}_{\mathrm{k}}\right| \mathrm{vs}$. $\mathrm{E}_{\mathrm{iR} \text {-free }}$ curve in (b) at a rotation speed of $3600 \mathrm{rpm}$ for comparison.

As mentioned before, HER on Pt in base is more sluggish than in acid and therefore diffusion limitation correction is less important. Here we show that diffusion correction is still necessary for obtaining accurate kinetics data. In a recent study, Rheinländer et al. argue that the HER branch only needs to be ohmically compensated to obtain kinetic HER currents on Pt in alkaline media, based on the observation that HER kinetic currents at different rotation speeds do not overlap when calculated using the irreversible Koutecky-Levich equation (Eq. 2), but do overlap when using the measured currents as the kinetic currents. ${ }^{10}$ In our analysis, we confirm that net-HER kinetic currents obtained by the irreversible Koutecky-Levich equation for different rotation speeds do not overlap (Figure 9a) however, net-HER kinetic currents extracted with the reversible Koutecky-Levich equation do overlap (Figure 9b).

Non-overlapping curves were obtained when $|\mathrm{i}|$ was plotted vs. $E-\eta_{d}$ at different rotation speed (Figure 9c), indicating that a diffusion overpotential correction using $\eta_{\mathrm{k}}=\eta-\eta_{\mathrm{d}}$ without a current correction is inadequate, as discussed in the previous section. Plots of $\left|i_{k}\right|$ (calculated with the modified Koutecky-Levich Eq. 35) vs. E $-\eta_{d}$ at different rotation speeds overlap completely for both HOR and HER branches (Figure 9d), demonstrating that this double correction method (both $\eta$ and i are corrected) is reliable in extracting kinetic information. Importantly, $\left|\mathrm{i}_{\mathrm{k}}\right|-\mathrm{E}$ plot obtained by correcting the current with the reversible Koutecky-Levich equation (Method 1, Figure $9 \mathrm{~b}$ ) matches those obtained with the double correction method (Method 2), demonstrating the consistency of the two approaches. The gray dashed curve and solid curves in Figure 9d are obtained following Methods 1 and 2, respectively, and they match well. The difference in potential range stems from the $\eta_{\mathrm{k}}=\eta-\eta_{\mathrm{d}}$ correction in Method 2, which modifies the potential range; whereas no potential correction is needed in Method 1.

It should be noted that the reversible Koutecky-Levich equation (Eq. 11) is only rigorously derived for one electron transfer elementary
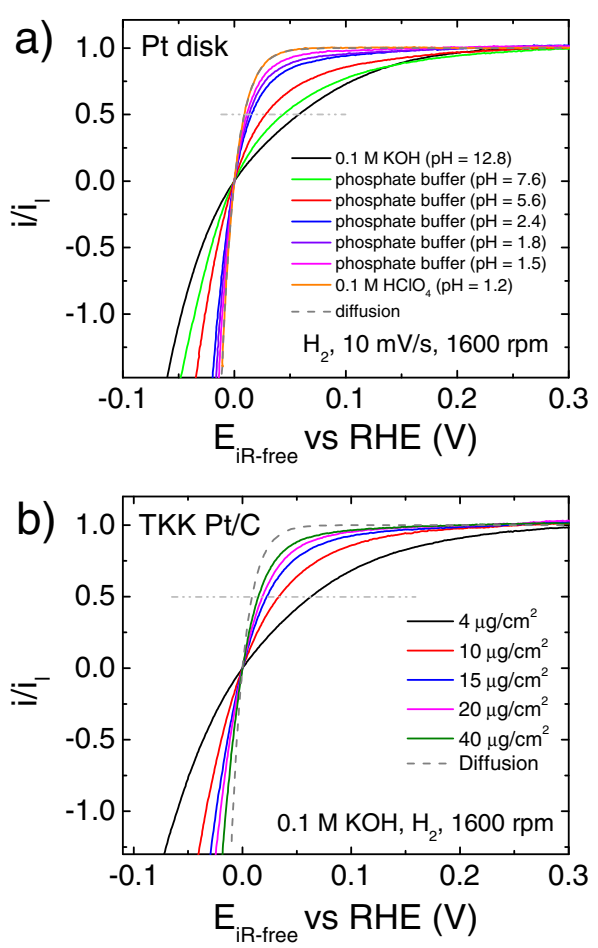

Figure 10. (a) HOR/HER polarization curves on $\mathrm{Pt}(\mathrm{pc})$ disk in $\mathrm{H}_{2}$-saturated $0.1 \mathrm{M} \mathrm{KOH}, 0.1 \mathrm{M} \mathrm{HClO}_{4}$ and phosphoric acid/phosphate buffer solutions with different $\mathrm{pHs}$, tested at a scanning rate of $10 \mathrm{mV} / \mathrm{s}$ and a rotation speed of $1600 \mathrm{rpm}$, (b) HOR/HER polarization curves on carbon supported Pt nanoparticles from TKK (Pt/C) with different Pt loadings tested in $\mathrm{H}_{2}$-saturated $0.1 \mathrm{M}$ $\mathrm{KOH}$, at $1 \mathrm{mV} / \mathrm{s}$ and $1600 \mathrm{rpm}$. 

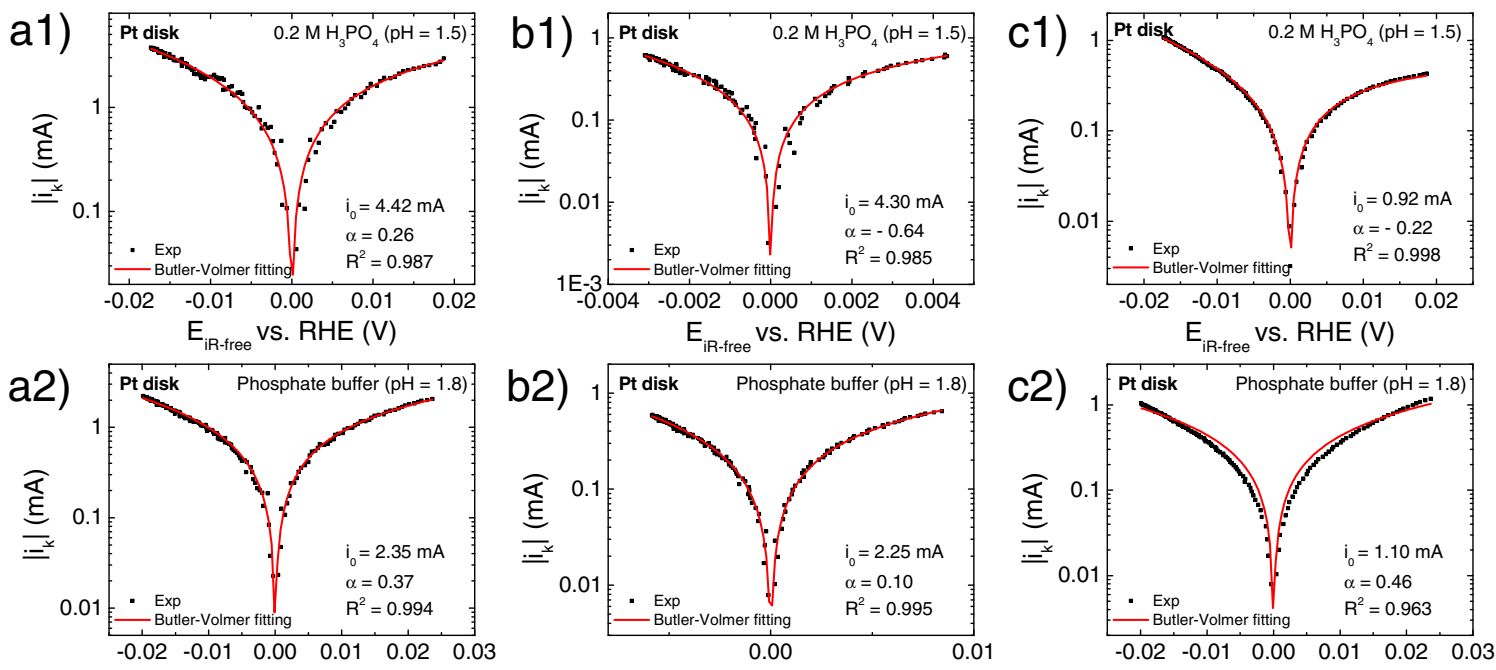

$E_{\text {iR-free }}$ vs. RHE (V)

$$
E_{\text {iR-free }} \text { vs. RHE (V) }
$$
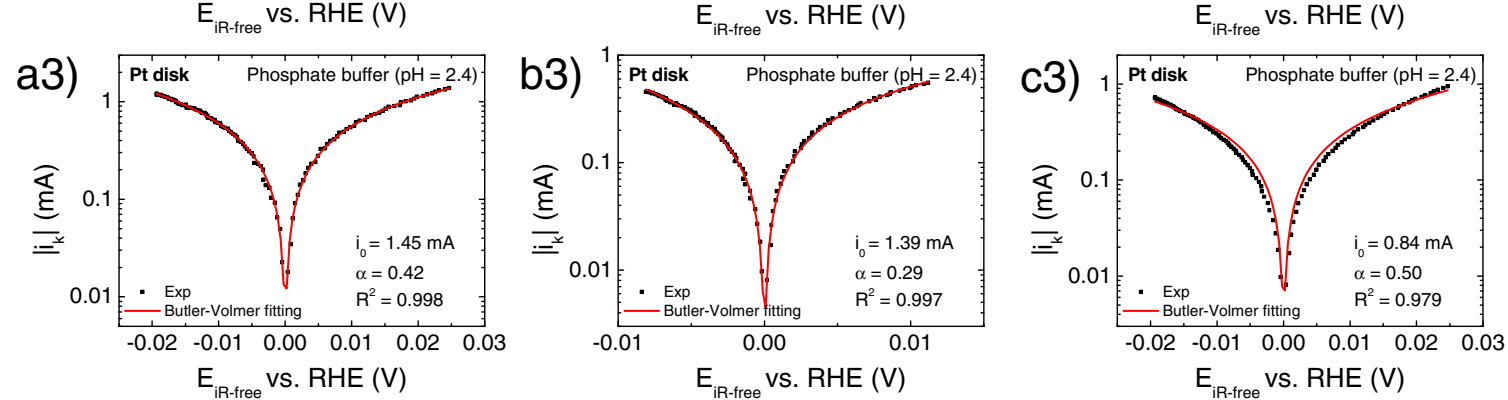

$E_{\text {iR-free }}$ vs. RHE (V)
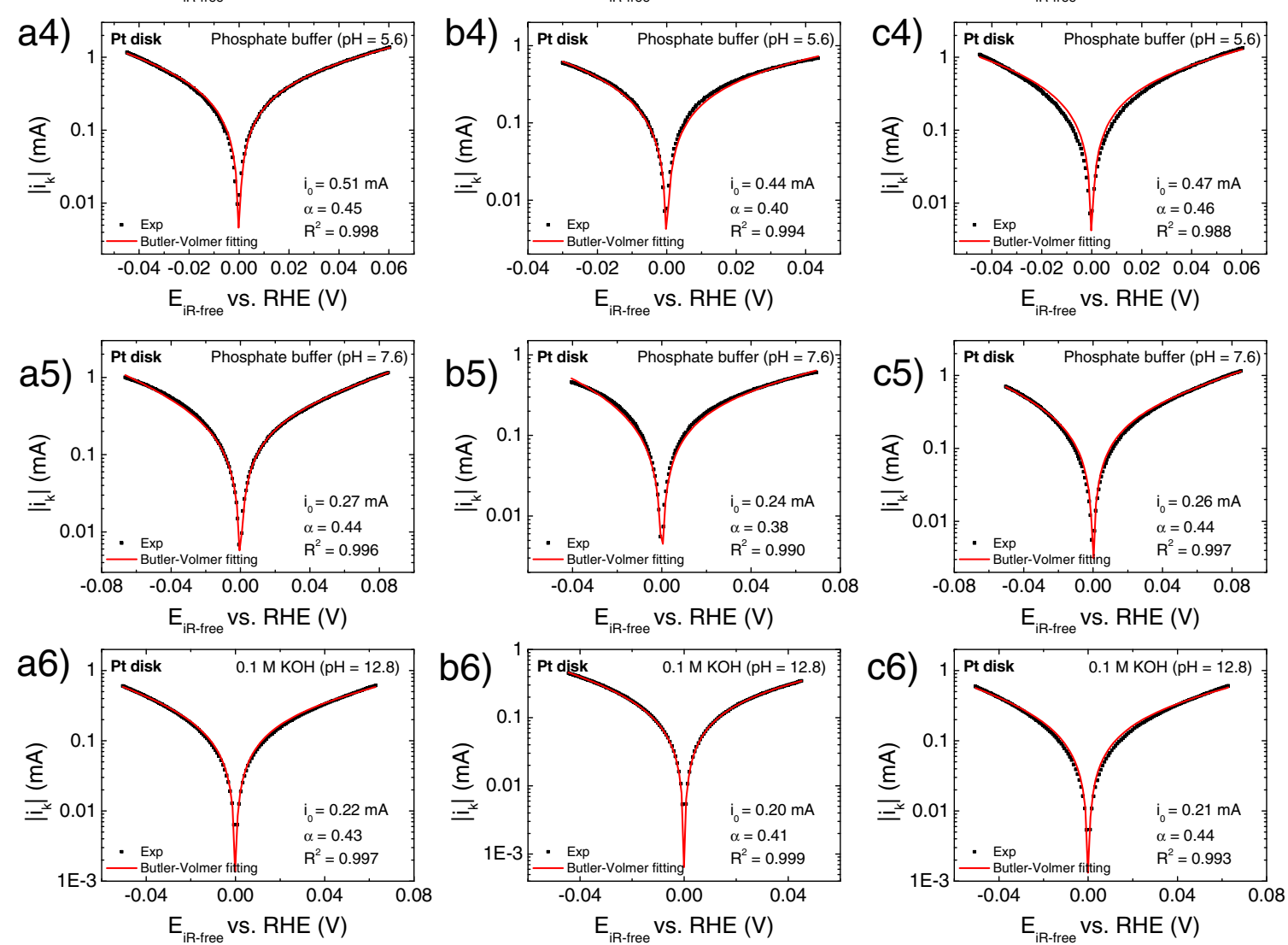

Figure 11. Tafel plots of HOR/HER kinetic currents and their corresponding fitting into a Butler-Volmer equation calculated from HOR/HER polarization curves measured on a Pt disk in electrolytes with different $\mathrm{pHs}$ at $10 \mathrm{mV} / \mathrm{s}$ and $1600 \mathrm{rpm}$, when (a1-a6) both HOR and HER kinetic currents were calculated using the reversible Koutecky-Levich equation $\left(1 / i=1 / i_{k}+1 / i_{d}\right)$, (b1-b6) both HOR and HER overpotentials are corrected by diffusion overpotential, and both HOR and HER currents were converted to kinetic currents using $i / i_{k}=\left(1-i / i_{l, a}\right)^{0.5}$, and (c1-c6) the HOR kinetic currents were calculated using irreversible Koutecky-Levich equation $\left(1 / i=1 / i_{k}+1 / i_{l}\right)$ and the HER kinetic currents were the measured ones corrected for internal resistance (iR). All overpotentials were iR-corrected. 
reactions. However, HOR/HER is inherently a two electron transfer reaction which consists of at least two elementary steps, e.g., TafelVolmer or Heyrovsky -Volmer, ${ }^{33}$

$$
\begin{gathered}
\text { Tafel : } H_{2}+2 * \leftrightarrow 2 H_{a d} \\
\text { Heyrovsky : } H_{2}+* \leftrightarrow H_{a d}+H^{+}+e \\
\text { Volmer : } H_{a d} \leftrightarrow H^{+}+e+*
\end{gathered}
$$

Regardless of the mechanism assumed, the reversible KouteckyLevich equation (Eq. 11) cannot be obtained. Additionally, discrepancy exists between the diffusion current equations derived for a one electron transfer - one step reaction $\left(\left(i_{d}=i_{l, a}\left(1-e^{-\frac{F \eta}{R T}}\right)\right.\right.$ from Eq. 16 when $i_{l, c}$ approaches infinity) and derived for HOR/HER $\left(i_{d}=i_{l, a}\left(1-e^{-\frac{2 F \eta}{R T}}\right)\right.$ in Eq. 5), which can be solved only if the summation of transfer coefficients for HOR and HER equals to 2. In this case, Eqs. 12 and 14 become Eqs. 41 and 42, the diffusion current will have the form of Eq. 5, and the reversible Koutecky-Levich equation (Eq. 11) remains valid. Similarly, the derivation of Method 2 also assumes the reaction involves only one electron transfer and one step reaction.

$$
\begin{gathered}
\frac{i}{i_{0}}=\left(1-\frac{i}{i_{l, a}}\right) e^{\alpha^{\prime} F \eta / R T}-e^{-\left(2-\alpha^{\prime}\right) F \eta / R T} \\
i_{k}=i_{0}\left(e^{\alpha^{\prime} F \eta / R T}-e^{-\left(2-\alpha^{\prime}\right) F \eta / R T}\right)
\end{gathered}
$$

The interdependence between reaction mechanism and kinetic parameters leads to a dilemma: electrokinetics, a powerful tool in the mechanistic study of multi-electron transfer reactions and the reliable extraction of kinetic information, e.g., $i_{0}$ and $\alpha$, predicates on the accurate knowledge of kinetic current. At the same time, the formula needed to deconvolute the kinetic and diffusional contributions to the measured currents is dependent on the knowledge of the same set of kinetic parameters, and in turn the reaction mechanism. One solution is to adopt techniques without mass transport limitation, such as the $\mathrm{H}_{2}$-pump method ${ }^{9,11,12}$ and the floating electrode method, ${ }^{17}$ to avoid the entanglement of kinetic and diffusional contributions. However, those methods typically require more complex setups and are often time consuming, and cannot evaluate materials in the disk forms. Another option is to derive an expression for an assumed mechanism with mass transport, and then fit experimental data numerically into the derived expression. ${ }^{14}$ While it is clearly more rigorous, given the large number of possible mechanisms for multiple electron transfer and multi-step processes, this method is more suitable for reaction systems with considerable existing knowledge and the number of possible mechanisms is limited. RDE method with its simplicity and reliability will remain a powerful technique in the mechanistic study of multi-electron and multi-step electrochemical processes provided that robust data analysis procedures are developed.

Although the reversible Koutecky-Levich equation cannot be rigorously derived for multi-electron, multistep electrochemical reactions, such as HER/HOR, the kinetic currents obtained is much more consistent than those obtained from irreversible Koutecky-Levich equation, as shown in Figures 6 and 7. The fundamental reason for the ability of reversible Koutecky-Levich equation to extract information for electrochemical reaction other than one-electron, one-step processes deserves further investigations. Our HOR/HER results suggest that it is reasonable to consider the reversible Koutecky-Levich equation as a semi-empirical expression to calculate kinetic currents with excellent accuracy.

Comparison of the three methods of calculating the kinetic current and the exchange current $\left(i_{0}\right)$ of HOR/HER. - The exchange current $\left(i_{0}\right)$ of HOR/HER can be obtained by fitting the kinetic current $\left(i_{k}\right)$ into the Butler-Volmer equation (Eq. 4) or fitting $i_{k}$ within a very small overpotential region into the linearized Butler-Volmer equation as in Eq. 43,

$$
i_{k}=i_{0} F \eta / R T
$$

Both approaches are based on sound theoretical ground and lead to similar $i_{0}$. The former method has the added benefit of yielding the transfer coefficient $\alpha$, which is related to the Tafel slope (TS) by TS $=2.303 \mathrm{RT} / \alpha \mathrm{F}$.

Experimentally, polarization curves with different exchange currents can be generated by varying the $\mathrm{pH}$ of the electrolyte, or the loading of the electrocatalyst: the exchange current increases as $\mathrm{pH}$ decreases or the loading increase. The HOR/HER polarization curve on a Pt disk approaches the concentration overpotential curve as the $\mathrm{pH}$ of the electrolyte decreases (Figure 10a). The following three methods were used for diffusion correction: 1) both HOR and HER currents are converted to kinetic currents by reversible Koutecky-Levich equation (Eq. 11) (Method 1), and 2) the overpotential is corrected by diffusion overpotential $\left(\eta_{\mathrm{k}}=\eta-\eta_{\mathrm{d}}\right)$ and current is converted to kinetic current by a modified Koutecky-Levich equation (Eq. 35) for both HOR and HER branches (Method 2) and 3) the net-HOR current is converted to kinetic currents by irreversible Koutecky-Levich equation (Eq. 2) while the HER current is uncorrected (Method 3). All the overpotentials were iR corrected. Tafel plots of HOR/HER kinetic currents at different $\mathrm{pHs}$ and their corresponding fittings with the Butler-Volmer equation were shown in Figure 11. The fittings with Method 1 (Figure 11a1-11a6) and Method 2 (Figure 11b1-11b6) are excellent in the $\mathrm{pH}$ range of 1.5-12.8, while fittings with Method 3 (Figure 11c1-11c6) become increasingly poor with decreasing $\mathrm{pHs}$, as the reaction is increasingly diffusion limited. The exchange current $\left(\mathrm{i}_{0}\right)$ determined

\begin{tabular}{|c|c|c|c|c|c|}
\hline \multicolumn{2}{|c|}{ Butler-Volmer } & \multirow{2}{*}{$\begin{array}{l}\text { Linear } \\
\mathrm{i}_{0}(\mathrm{~mA})\end{array}$} & \multicolumn{2}{|c|}{ Butler-Volmer } & \multirow{2}{*}{$\begin{array}{l}\text { Linear } \\
\mathrm{i}_{0}(\mathrm{~mA})\end{array}$} \\
\hline $\mathrm{i}_{0}(\mathrm{~mA})$ & $\alpha$ & & $\mathrm{i}_{0}(\mathrm{~mA})$ & $\alpha$ & \\
\hline 4.30 & -0.64 & 4.45 & 0.92 & -0.22 & 0.90 \\
\hline 2.25 & 0.1 & 2.33 & 1.10 & 0.46 & 0.91 \\
\hline 1.39 & 0.29 & 1.42 & 0.84 & 0.50 & 0.74 \\
\hline 0.44 & 0.40 & 0.49 & 0.47 & 0.46 & 0.40 \\
\hline 0.24 & 0.38 & 0.27 & 0.26 & 0.44 & 0.24 \\
\hline 0.20 & 0.41 & 0.20 & 0.21 & 0.44 & 0.19 \\
\hline
\end{tabular}

Table I. Comparison of exchange currents $\left(i_{0}\right)$ from three different diffusion-correction methods for HOR/HER on a $5 \mathrm{~mm}$ diameter Pt disk in electrolytes with different pHs.

Method $1^{\mathrm{a}}$

\begin{tabular}{lllll} 
& & \multicolumn{2}{c}{ Butler-Volmer } & \multirow{2}{*}{ Linear } \\
Electrolyte & $\mathrm{pH}$ & $\mathrm{i}_{0}(\mathrm{~mA})$ & $\alpha$ & $\mathrm{i}_{0}(\mathrm{~mA})$ \\
\hline $0.2 \mathrm{M} \mathrm{H}_{3} \mathrm{PO}_{4}$ & 1.5 & 4.42 & 0.26 & 4.61 \\
Phosphoric acid/phosphate buffer & 1.8 & 2.35 & 0.37 & 2.38 \\
& 2.4 & 1.45 & 0.42 & 1.49 \\
& 5.6 & 0.51 & 0.45 & 0.50 \\
$0.1 \mathrm{M} \mathrm{KOH}$ & 7.6 & 0.27 & 0.44 & 0.28 \\
& 12.8 & 0.22 & 0.43 & 0.21
\end{tabular}

Method $2^{\mathrm{b}}$

${ }^{\mathrm{a}}$ The kinetic current $\left(\mathrm{i}_{\mathrm{k}}\right)$ for both HOR and HER branch is calculated by reversible Koutecky-Levich equation $\frac{1}{i}=\frac{1}{i_{k}}+\frac{1}{i_{d}}$.

${ }^{\mathrm{b}}$ The overpotential is corrected by diffusion overpotential $\eta_{\mathrm{k}}=\eta-\eta_{\mathrm{d}}$, while the measured current is converted to kinetic current by $\frac{i}{i_{k}}=\left(1-\frac{i}{i_{l, a}}\right)^{0.5}$.

${ }^{\mathrm{c}}$ The kinetic current $\left(\mathrm{i}_{\mathrm{k}}\right)$ for HOR branch is calculated by irreversible Koutecky-Levich equation $\frac{1}{i}=\frac{1}{i_{k}}+\frac{1}{i_{l}}$ while the kinetic current ( $\mathrm{i}_{\mathrm{k}}$ ) for HER branch remains uncorrected. 

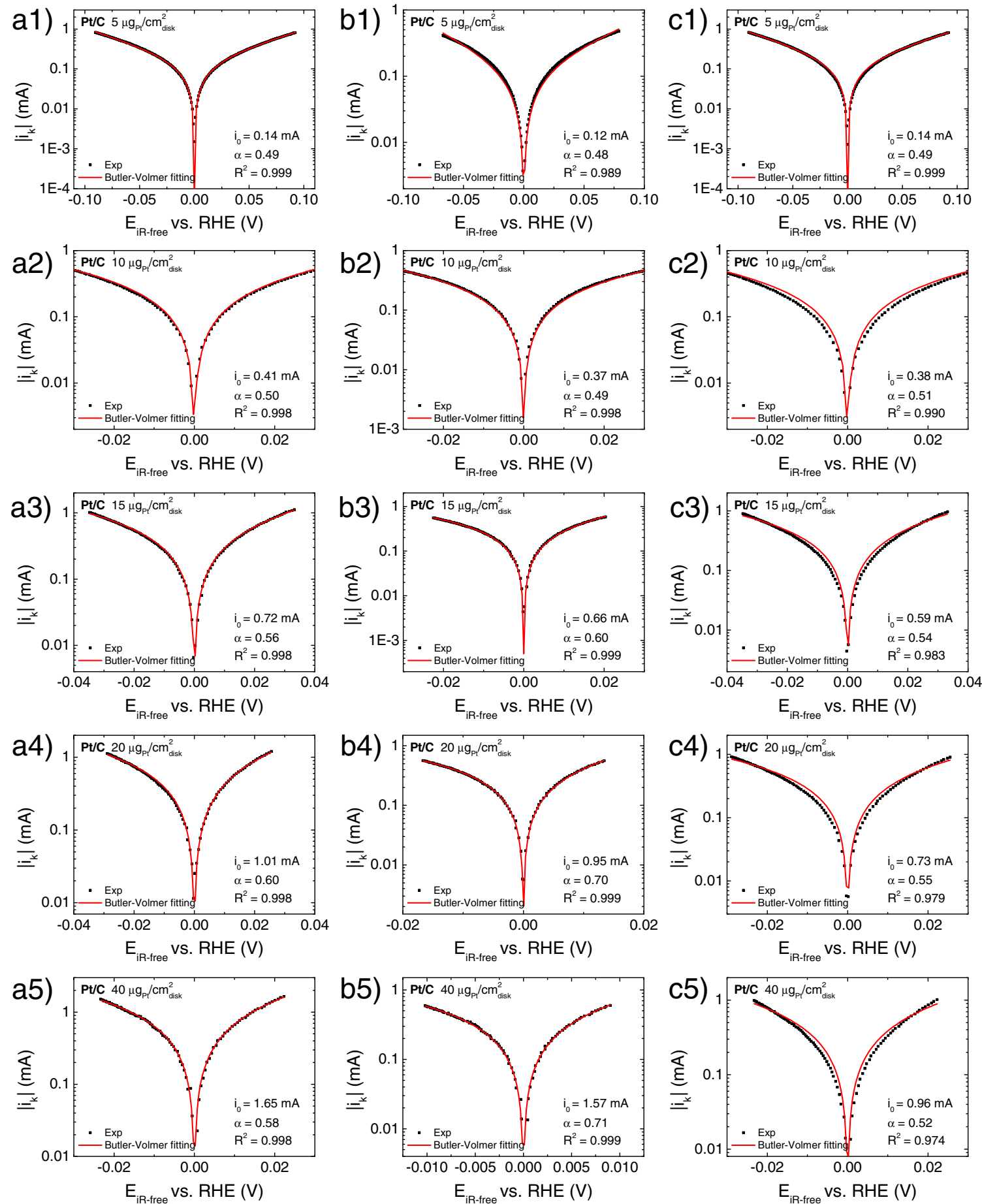

Figure 12. Tafel plots of HOR/HER kinetic currents and their corresponding fitting into a Butler-Volmer equation calculated from HOR/HER polarization curves measured on Pt/C in $0.1 \mathrm{M} \mathrm{KOH}$ with Pt loadings from 4 to $40 \mu \mathrm{g}_{\mathrm{Pt}} / \mathrm{cm}^{2}$ disk at $1 \mathrm{mV} / \mathrm{s}$ and $1600 \mathrm{rpm}$, when (a1-a5) both HOR and HER kinetic currents were calculated using the reversible Koutecky-Levich equation $\left(1 / i=1 / i_{k}+1 / i_{d}\right)$, (b1-b5) both HOR and HER overpotentials are corrected by diffusion overpotential, and both HOR and HER currents were converted to kinetic currents using $i / i_{k}=\left(1-i / i_{l, a}\right)^{0.5}$, and (c1-c5) the HOR kinetic currents were calculated using irreversible Koutecky-Levich equation $\left(1 / i=1 / i_{k}+1 / i_{l}\right)$ and the HER kinetic currents were the measured ones corrected for internal resistance (iR). All overpotentials were iR-corrected.

from the three methods mentioned above increases as the $\mathrm{pH}$ of the electrolyte decreases (Table I). $\mathrm{i}_{0}$ determined from Butler-Volmer fitting and linear fitting are similar for all methods. The $\mathrm{i}_{0}$ from Methods 1 and 2 are identical within the experimental errors, while the $i_{0}$ from Method 3 only agrees with the rest two at high $\mathrm{pHs}$ when the electrode kinetics is slow, and is substantially underestimated at lower $\mathrm{pHs}$ when the kinetics becomes faster (Table I). Transfer coefficients $(\alpha)$ from
Method 1 are more meaningful compared with those obtained from Method 2, as an $\alpha$ value of 0.5 is assumed in the derivation of Method 2 (the negative value at $\mathrm{pH}=1.5$ is clearly not physical, Table I).

HOR/HER polarization curves on carbon supported Pt nanoparticles approach the concentration overpotential curve as the loading of Pt increases from 4 to $40 \mu \mathrm{g}_{\mathrm{Pt}} / \mathrm{cm}^{2}{ }_{\text {disk }}$ (Figure 10b), and the Butler-Volmer fittings of the HOR/HER kinetic currents obtained 
Table II. Comparison of exchange current $\left(i_{0}\right)$, transfer coefficient $(\alpha)$ and exchange current density $\left(j_{0}\right)$ from three different diffusion-correction methods for HOR/HER on a $5 \mathrm{~mm}$ diameter glassy carbon electrode with different Pt loadings in $0.1 \mathrm{M} \mathrm{KOH}$ at $1600 \mathrm{rpm}$.

\begin{tabular}{|c|c|c|c|c|c|c|c|c|c|c|c|}
\hline \multirow[b]{2}{*}{ Loading $\left(\mu \mathrm{g} / \mathrm{cm}^{2}{ }_{\text {disk }}\right)$} & \multirow[b]{2}{*}{ Surface area $\left(\mathrm{cm}^{2}\right)$} & \multirow[b]{2}{*}{$\operatorname{ECSA}\left(\mathrm{m}^{2} / \mathrm{g}\right)$} & \multicolumn{3}{|c|}{ Method $1^{\mathrm{a}}$} & \multicolumn{3}{|c|}{ Method $2^{\mathrm{b}}$} & \multicolumn{3}{|c|}{ Method $3^{c}$} \\
\hline & & & $\mathrm{i}_{0}(\mathrm{~mA})$ & $\alpha$ & $\mathrm{j}_{0}\left(\mathrm{~mA} / \mathrm{cm}^{2}{ }_{\mathrm{Pt}}\right)^{\mathrm{d}}$ & $\mathrm{i}_{0}(\mathrm{~mA})$ & $\alpha$ & $\mathrm{j}_{0}\left(\mathrm{~mA} / \mathrm{cm}^{2}{ }_{\mathrm{Pt}}\right)$ & $\mathrm{i}_{0}(\mathrm{~mA})$ & $\alpha$ & $\mathrm{j}_{0}\left(\mathrm{~mA} / \mathrm{cm}^{2} \mathrm{Pt}\right)$ \\
\hline 4 & 0.35 & 45 & 0.14 & 0.49 & 0.40 & 0.12 & 0.48 & 0.34 & 0.14 & 0.49 & 0.39 \\
\hline 10 & 1.03 & 53 & 0.41 & 0.50 & 0.40 & 0.37 & 0.49 & 0.35 & 0.38 & 0.51 & 0.37 \\
\hline 15 & 1.60 & 54 & 0.72 & 0.56 & 0.45 & 0.66 & 0.60 & 0.41 & 0.59 & 0.54 & 0.37 \\
\hline 20 & 2.20 & 56 & 1.01 & 0.60 & 0.46 & 0.95 & 0.70 & 0.43 & 0.73 & 0.55 & 0.33 \\
\hline 40 & 4.00 & 51 & 1.65 & 0.58 & 0.41 & 1.57 & 0.70 & 0.39 & 0.96 & 0.52 & 0.24 \\
\hline
\end{tabular}

${ }^{\mathrm{a}}$ The kinetic current $\left(\mathrm{i}_{\mathrm{k}}\right.$ ) for both HOR and HER branch is calculated by reversible Koutecky-Levich equation $\frac{1}{i}=\frac{1}{i_{k}}+\frac{1}{i_{d}}$.

${ }^{\mathrm{b}}$ The overpotential is corrected by diffusion overpotential $\eta_{\mathrm{k}}=\eta-\eta_{\mathrm{d}}$, while the measured current is converted to kinetic current by $\frac{i}{i_{k}}=\left(1-\frac{i}{i_{l, a}}\right)^{0.5}$.

${ }^{\mathrm{c}}$ The kinetic current $\left(\mathrm{i}_{\mathrm{k}}\right)$ for HOR branch is calculated by irreversible Koutecky-Levich equation $\frac{1}{i}=\frac{1}{i_{k}}+\frac{1}{i_{l}}$ while the kinetic current (i $\mathrm{i}_{\mathrm{k}}$ ) for HER branch remains uncorrected.

${ }^{\mathrm{d}}$ Similar current densities for samples with different metal loadings prove that the diffusion in the Pt catalyst layer does not affect the HOR/HER rates.

using Method 3 are worse than those obtained using Methods 1 and 2 (Figure 12). The $\mathrm{i}_{0}$ determined from the three methods are similar to each other when the loading is less than $15 \mu \mathrm{g}_{\mathrm{Pt}} / \mathrm{cm}^{2}{ }_{\text {disk }}$ or $\mathrm{i}_{0} / \mathrm{i}_{1} \approx$ $1.5\left(i_{l}\right.$ is the limiting current in the HOR branch, its value is about $2.5 \mathrm{~mA} / \mathrm{cm}^{2}$ disk at $\left.1600 \mathrm{rpm}\right)$, however, the $\mathrm{i}_{0}$ determined from Method 3 are significantly underestimated as the loading of the electrocatalyst grows and the reaction becomes increasingly diffusion limited (Table II), which is consistent with the results obtained on the $\mathrm{Pt}$ disk in the previous section. For HOR/HER, using the irreversible Koutecky-Levich equation to determine $j_{k}$ yields reasonable results only when the total exchange current is less than $1.5 \mathrm{i}_{1}$ (as in the case of $\mathrm{HOR} / \mathrm{HER}$ on a Pt disk at different $\mathrm{pHs}$ or $\mathrm{Pt} / \mathrm{C}$ with a relatively low loading in $0.1 \mathrm{M} \mathrm{KOH}$ ), and substantially underestimated exchange current densities are obtained as the true exchange current becomes larger. Therefore, Methods 1 and 2 are recommended in calculating kinetic currents and exchange current densities for HOR/HER. Method 1 is preferred because it requires less correction and Method 2 requires prior knowledge of $\alpha$ (assumed to be 0.5 in the derivation) to calculate $i_{k}$ at overpotential $\eta-\eta_{d}$.

\section{Conclusions}

Our study shows that HER is diffusion limited when the electrode kinetics is facile. The diffusion limitation behavior of HER originates from the slow mass transport of produced $\mathrm{H}_{2}$ on the electrode to the bulk electrolyte and the reversible nature of HOR/HER. Although the diffusion overpotential for HER is typically small, it could have a significant impact on the kinetic analysis especially in the low overpotential region, leading to underestimated activities and inaccurate mechanistic interpretations. The reversible nature of HOR/HER dictates that the reversible Koutecky-Levich equation should be used to calculate kinetic current while the use of the irreversible KouteckyLevich equation to obtain kinetic currents can lead to significant errors when the electrode kinetics is facile and the process is partially diffusion controlled. In order to obtain accurate kinetic information from polarization curves using RDE method, we recommend: 1) perform $\mathrm{iR}$ correction to compensate the solution resistance; and 2) correct the measured current with the reversible Koutecky-Levich equation to obtain the kinetic current.

\section{Acknowledgment}

This work is supported by the US Department of Energy ARPAE Program (DE-AR0000009) and University of Delaware Research Foundation (CCST452124)

\section{References}

1. H. A. Gasteiger, N. M. Markovic, and P. N. Ross, The Journal of Physical Chemistry, 99, 8290 (1995).
2. T. J. Schmidt, H. A. Gasteiger, G. D. Stäb, P. M. Urban, D. M. Kolb, and R. J. Behm, Journal of The Electrochemical Society, 145, 2354 (1998).

3. W. Sheng, H. A. Gasteiger, and Y. Shao-Horn, Journal of The Electrochemical Society, 157, B1529 (2010).

4. D. R. Lawson, L. D. Whiteley, C. R. Martin, M. N. Szentirmay, and J. I. Song, Journal of The Electrochemical Society, 135, 2247 (1988).

5. F. Gloaguen, F. Andolfatto, R. Durand, and P. Ozil, Journal of Applied Electrochemistry, 24, 863 (1994)

6. Z. Galus, C. Olson, H. Y. Lee, and R. N. Adams, Analytical Chemistry, 34, 164 (1962).

7. H. A. Gasteiger, S. S. Kocha, B. Sompalli, and F. T. Wagner, Applied Catalysis B: Environmental, 56, 9 (2005).

8. K. J. J. Mayrhofer, D. Strmcnik, B. B. Blizanac, V. Stamenkovic, M. Arenz, and N. M. Markovic, Electrochimica Acta, 53, 3181 (2008).

9. J. Durst, C. Simon, F. Hasché, and H. A. Gasteiger, Journal of The Electrochemical Society, 162, F190 (2015).

10. P. J. Rheinländer, J. Herranz, J. Durst, and H. A. Gasteiger, Journal of The Electrochemical Society, 161, F1448 (2014).

11. K. C. Neyerlin, W. Gu, J. Jorne, and H. A. Gasteiger, Journal of The Electrochemical Society, 154, B631 (2007).

12. J. Durst, A. Siebel, C. Simon, F. Hasche, J. Herranz, and H. A. Gasteiger, Energy \& Environmental Science, 7, 2255 (2014).

13. V. S. Bagotzky and N. V. Osetrova, Journal of Electroanalytical Chemistry and Interfacial Electrochemistry, 43, 233 (1973).

14. S. Chen and A. Kucernak, The Journal of Physical Chemistry B, 108, 13984 (2004).

15. J. Zhou, Y. Zu, and A. J. Bard, Journal of Electroanalytical Chemistry, 491, 22 (2000).

16. C. G. Zoski, The Journal of Physical Chemistry B, 107, 6401 (2003).

17. C. M. Zalitis, D. Kramer, and A. R. Kucernak, Physical Chemistry Chemical Physics, 15, 4329 (2013)

18. L. Cheng, W. Huang, Q. Gong, C. Liu, Z. Liu, Y. Li, and H. Dai, Angewandte Chemie International Edition, 53, 7860 (2014).

19. J. Deng, P. Ren, D. Deng, L. Yu, F. Yang, and X. Bao, Energy \& Environmental Science, 7, 1919 (2014).

20. M. S. Faber, R. Dziedzic, M. A. Lukowski, N. S. Kaiser, Q. Ding, and S. Jin, Journal of the American Chemical Society, 136, 10053 (2014).

21. M.-R. Gao, J.-X. Liang, Y.-R. Zheng, Y.-F. Xu, J. Jiang, Q. Gao, J. Li, and S.-H. Yu, Nat Commun, $\mathbf{6}$ (2015).

22. M. Tavakkoli, T. Kallio, O. Reynaud, A. G. Nasibulin, C. Johans, J. Sainio, H. Jiang, E. I. Kauppinen, and K. Laasonen, Angewandte Chemie International Edition, 54, 4535 (2015).

23. D.-Y. Wang, M. Gong, H.-L. Chou, C.-J. Pan, H.-A. Chen, Y. Wu, M.-C. Lin, M. Guan, J. Yang, C.-W. Chen, Y.-L. Wang, B.-J. Hwang, C.-C. Chen, and H. Dai, Journal of the American Chemical Society, 137, 1587 (2015).

24. N. M. Marković, B. N. Grgur, and P. N. Ross, The Journal of Physical Chemistry B, 101, 5405 (1997).

25. J. Maruyama, M. Inaba, K. Katakura, Z. Ogumi, and Z.-i. Takehara, Journal of Electroanalytical Chemistry, 447, 201 (1998).

26. J. Durst, C. Simon, A. Siebel, P. J. Rheinländer, T. Schuler, M. Hanzlik, J. Herranz, F. Hasché, and H. A. Gasteiger, ECS Transactions, 64, 1069 (2014).

27. J. Zheng, Z. Zhuang, B. Xu, and Y. Yan, ACS Catalysis, 4449 (2015).

28. Q. Chen, L. Luo, and H. S. White, Langmuir, 31, 4573 (2015).

29. Q. Chen, L. Luo, H. Faraji, S. W. Feldberg, and H. S. White, The Journal of Physical Chemistry Letters, 5, 3539 (2014).

30. A. J. Bard and L. R. Faulkner, Electrochemical Methods: Fundamentals and Applications, p. 864, John Wiley \& Sons, Inc (2001).

31. W. Sheng, A. P. Bivens, M. Myint, Z. Zhuang, R. V. Forest, Q. Fang, J. G. Chen, and Y. Yan, Energy \& Environmental Science, 7, 1719 (2014).

32. W. Sheng, Z. Zhuang, M. Gao, J. Zheng, J. G. Chen, and Y. Yan, Nat Commun, 6 (2015).

33. M. W. Breiter, in Handbook of Fuel Cells, p. 361, John Wiley \& Sons, Ltd (2010). 